\title{
Technical Note: Interference errors in infrared remote sounding of the atmosphere
}

\author{
R. Sussmann and T. Borsdorff \\ IMK-IFU, Forschungszentrum Karlsruhe, Kreuzeckbahnstrasse 19, 82467 Garmisch-Partenkirchen, Germany \\ Received: 13 November 2006 - Published in Atmos. Chem. Phys. Discuss.: 12 December 2006 \\ Revised: 26 March 2007 - Accepted: 31 May 2007 - Published: 6 July 2007
}

\begin{abstract}
Classical error analysis in remote sounding distinguishes between four classes: "smoothing errors," "model parameter errors," "forward model errors," and "retrieval noise errors". For infrared sounding "interference errors", which, in general, cannot be described by these four terms, can be significant. Interference errors originate from spectral residuals due to "interfering species" whose spectral features overlap with the signatures of the target species. A general method for quantification of interference errors is presented, which covers all possible algorithmic implementations, i.e., fine-grid retrievals of the interfering species or coarse-grid retrievals, and cases where the interfering species are not retrieved. In classical retrieval setups interference errors can exceed smoothing errors and can vary by orders of magnitude due to state dependency. An optimum strategy is suggested which practically eliminates interference errors by systematically minimizing the regularization strength applied to joint profile retrieval of the interfering species. This leads to an interfering-species selective deweighting of the retrieval. Details of microwindow selection are no longer critical for this optimum retrieval and widened microwindows even lead to reduced overall (smoothing and interference) errors. Since computational power will increase, more and more operational algorithms will be able to utilize this optimum strategy in the future. The findings of this paper can be applied to soundings of all infrared-active atmospheric species, which include more than two dozen different gases relevant to climate and ozone. This holds for all kinds of infrared remote sounding systems, i.e., retrievals from ground-based, balloon-borne, airborne, or satellite spectroradiometers.
\end{abstract}

\section{Introduction}

During the last decade, more and more, infrared remote sounding measurements have been used to obtain profiles

Correspondence to: R. Sussmann

(ralf.sussmann@imk.fzk.de) of atmospheric composition and temperature from ground or space. Infrared profiling techniques complement the older microwave profilers in many ways, e.g., with respect to the altitude range attainable and the atmospheric trace species under consideration. The theoretical framework for the retrieval of profiles from spectral measurements via optimal estimation was developed three decades ago (Rodgers, 1976) and was applied to microwave soundings in the beginning. Later, a concept for the error analysis was formulated (Rodgers, 1990, 2000; Connor et al., 1995) to distinguish between four different classes of errors, i.e., "smoothing errors", "model parameter errors", "forward model errors", and "retrieval noise errors". This classical error analysis has also been applied to infrared retrievals. However, in the infrared, a very frequently encountered problem in retrieving the "target quantity" is due to "interfering species". This occurs because the vibration-rotation bands of different species often overlap in the infrared atmospheric spectrum. Keeping in mind that the wings of (infrared) spectral lines always expand asymptotically towards plus-minus infinity in the frequency domain, it becomes clear that individual spectral lines used for the profile retrieval of an atmospheric target species, in principle, always overlap with neighboring spectral lines of other interfering species. This leads to "interference errors" that can, in general, not be treated by either of the above mentioned four terms of classical error analysis.

Throughout this paper the term "interference error" refers to all errors that originate from any type of soft or hard constraint imposed on the retrieval of the profile of an interfering species in an algorithm; this causes spectral residuals (measured minus simulated) around the spectral signature of the interfering species. In consequence, the profile retrieval of the target species tends to compensate for this, meaning that an artifact is introduced into the retrieved target profile.

In addition to the described effect from interfering species we will also include in the term "interference error" all errors from constraints imposed on the retrieval of any additional,

Published by Copernicus Publications on behalf of the European Geosciences Union. 
varying, vector-type physical quantity which impacts the measurement vector. One example are temperature profiles, which are retrieved jointly with the target species in some retrieval setups in order to minimize errors from insufficient knowledge of the true temperature profile at the time of observation. For simplicity, we will hereafter extend the term "interfering species" to include these additional interfering vectors.

It is also important to specify, what our concept of interference errors does not mean throughout this paper. It does not have to do with any intrinsic error in the forward modeling, i.e., either with errors in the forward model parameters, such as errors in the spectroscopic parameters of interfering species, or with errors in the forward model itself like errors in line shape modeling. Both kinds of errors also lead to residuals in the retrieval of the interfering species and thereby introduce errors to the retrieval of the target species, and, therefore, might also be entitled "interference errors". However, these two error classes can be clearly attributed to and treated as "model parameter errors" (above example of errors in spectroscopic parameters) and to "forward model errors" (above example of errors in line shape modeling), and we will not further treat them within this paper.

This being said we like to point to an exception, i.e., a special case where interference errors in the sense of our paper can in fact be treated by the existing concept of "model parameter errors" as defined in Rodgers (2000, Eq. 3.16, second term): interfering species are sometimes not retrieved due to computation power limitations, e.g., in satellite infrared remote sensing. Interference errors originate in this case from a different effect. They do not arise from any constraint to the retrieval of interfering species (because they are not retrieved), nor from intrinsic forward model or model parameter errors, but from neglecting the likely discrepancies between the true profile of an interfering species at the moment of observation and its fixed a priori profile used for forward modeling. This again leads to spectral residuals that introduce errors to the retrieval of the target species. Formally, the discrepancies between the true profile of an interfering species and the one used for forward modeling can be treated by the concept of model parameter errors. This kind of error quantification for the case of unretrieved interfering species has been performed previously, and a minimization of interference errors in this case was achieved by optimized microwindow selection, i.e., an extensive microwindow cutting aiming at a minimized inclusion of spectral signatures of interfering species while preserving the main features of the target species (e.g., von Clarmann and Echle, 1998; Echle et al., 2000; Dudhia et al., 2002).

An alternative approach is to jointly retrieve the interfering species and the target species at the same time. This leads to a reduced interference effect compared to the case of unretrieved interfering species for a given microwindow set. This is achieved at the cost of increased computation time, however. A mathematical formulation for calculating an interfer- ence error covariance due to the joint retrieval of interfering species has been given for the first time by Rodgers and Connor (2003, Eq. 8 therein). This formulation holds for characterization of algorithms using a retrieval grid for the interfering species that is fine enough to sufficiently describe the vertical variations of their profiles. Applications of Eq. (8) as given by Rodgers and Connor (2003) for this special case of joint fine-grid retrievals of interfering species have been shown by Worden et al. (2004) and Bowman et al. (2006).

The goal of this paper is to maturate the approach of jointly retrieving the interfering species with a focus on two major issues, namely i) how to generalize interference error quantification to make it applicable to all different practical algorithmic implementations (i.e., fine-grid retrievals of interfering species, coarse-grid retrievals of interfering species, and unretrieved interfering species), and ii) how to systematically minimize interference errors.

Ad i). A direct application of Eq. (8) given in Rodgers and Connor (2003) to algorithms using coarse-grid retrievals of interfering species leads to significant underestimations of interference errors in case the true atmospheric profile variability of the interfering species is not adequately represented by the coarse retrieval grid. Therefore, we suggest a generalization of this formulation in order to enable interference error characterization not only for fine-grid retrievals of the interfering species but also for coarse-grid retrievals. The generalization includes a distinction between jointly retrieved scalars (e.g., frequency shift) and interfering vectors (e.g., describing interfering species which are subject to profiletype variability in the atmosphere). For all interfering vectors a sufficiently fine retrieval grid is implemented in order to be able to map an estimate of their true atmospheric covariance into the error analysis. Finally, the coarse retrieval grid of the standard retrieval to be characterized is emulated on the fine grid via a dedicated block-type soft constraint. (This generalization includes at the same time the option to characterize errors from unretrieved interfering species, formally in retrieval space, by applying an infinitely strong soft constraint. This may be more easily implemented than the approach using the classical concept of model parameter errors, see above).

Ad ii). This paper shows that interference errors can still become comparable to or larger than smoothing errors in case of standard retrievals where the interfering species are jointly retrieved together with the target species. To overcome this situation, we suggest an optimum strategy for setting up the regularization matrices used for retrieval of the interfering species. It allows to minimize interference errors to become negligible compared to other significant error sources like the smoothing error. It will be shown that details of microwindow selection are no longer critical for this optimum retrieval and widened microwindows even lead to reduced overall (smoothing and interference) errors.

Interference errors due to retrieved interfering species as discussed in this paper are different, but in a sense related 
to "smoothing errors", since for their quantification the regularization matrix of the retrieval of the interfering species as well as an estimate of their true covariance has to be known (in case of smoothing errors: regularization matrix and covariance of the target species). Therefore we discuss in this paper the significance of interference errors in terms of their magnitude relative to smoothing errors.

Appendix A gives definitions and a recapitulation of the classical error analysis of remote sounding according to Rodgers (2000). Section 2 is the central part of this paper and describes the general theoretical formulation to quantify interference errors: Case I treats algorithms using a finegrid profile retrieval of the interfering species, Case II treats coarse-grid retrievals of the interfering species, and Case III deals with cases where the interfering species are not retrieved. Section 2.4 discusses the factors influencing interference errors and presents an optimum strategy to practically eliminate interference errors. In Sect. 3 our quantification method is illustrated by applying it to the standard approach of optimal estimation of $\mathrm{CO}$ profiles from a test ensemble of ground-based solar spectra recorded with the high-resolution Fourier-transform spectrometer at the NDACC (Network for the Detection of Atmospheric Composition Change) Primary Station Zugspitze, Germany (see, e.g., Sussmann and Schäfer, 1997; Sussmann et al., 2005a, b). Section 4 presents case studies for solar CO retrievals, showing different impacts upon the interference errors which are due to varied constraints applied for retrieval of the interfering species (including the optimum strategy) as well as varied microwindows. Finally, Sect. 5 presents a summary and some of the conclusions which can be drawn from this.

\section{General formulation for quantification of interference errors}

Our general formulation of interference errors is an extension of Rogers' (1990, 2000) formulation of error analysis for remote sounding. A brief recapitulation is therefore given in Appendix A.

In Sect. 2.1 we will show that a new class of errors, i.e., one in addition to the four classes of Eq. (A1), arises if we define a generalized state vector including not only the target profile, but also all further retrieval parameters. The effect is a split up of the first term of Eq. (A1) into the smoothing error plus additional terms, which we will call interference errors.

The quantification of interference errors in Sect. 2.2 requires a different treatment for 3 cases which are distinguished by the type of constraint imposed on the interfering species within an operational retrieval algorithm.

Case I (Sect. 2.2.1) introduces the formulation for quantification of interference errors in the (rare) case that the interfering species are retrieved as profiles on a retrieval grid that is fine enough so that their true high resolution atmo- spheric profile variability can be properly mapped into the error analysis.

Case II (Sect. 2.2.2) describes algorithms using a coarsegrid retrieval of the interfering species. We draw attention to the critical point that in this case a direct application of the Case I formulation yields to erroneous results. The correct interference error analysis for Case II requires first an emulation of the coarse-grid retrieval on a sufficiently fine grid which then allows for subsequent application of the Case I formalism on the fine grid.

Case III describes algorithms where the interfering species are not retrieved. Section 2.2.3 discusses two different methods of quantifying interference errors in this case.

\subsection{Generalized state vector}

We re-define $\boldsymbol{x} \in \mathfrak{R}^{l}$ to be a "generalized state vector" which takes into account all the parameters to be retrieved, not only the atmospheric target profile, see Eq. (1). We will refer to the part of $\boldsymbol{x}$ describing the atmospheric target profile to be retrieved as $t \in \mathfrak{R}^{n}$. It contains, for example, scaling factors for layer-averaged volume mixing ratios (VMR) of an a priori profile of the target species, on a grid of $n=100$ layers each $1 \mathrm{~km}$ thick, covering the vertical range between 0 and $100 \mathrm{~km}$ altitude. The remaining sub-vector of $\boldsymbol{x}$ represents all further parameters to be retrieved in addition to the target parameters. It comprises vectors $\boldsymbol{v}_{1}, \boldsymbol{v}_{2}, \ldots$ with length $n$, describing the profiles of interfering species, i.e., species different from the target species which show spectral signatures within the microwindows as well as additional vector-type quantities that are retrieved, such as temperature profiles (which can also cause an interference effect). Furthermore, it consists of scalar-type retrieval parameters $s_{1}, s_{2}, \ldots$, denoted as "retrieved auxiliary scalar parameters" hereafter. These parameters are candidates for forward model parameters. They are, however, retrieved because they are not known accurately enough (e.g., a frequency shift between the measured and simulated spectrum). Note that we introduce the additional class "retrieved auxiliary scalar parameters" since, according to the nomenclature of Rodgers (1976), "forward model parameters" are fixed parameters that are not being retrieved. 


$$
\boldsymbol{x}=\left(\begin{array}{c}
\boldsymbol{t} \\
\boldsymbol{v}_{1} \\
\boldsymbol{v}_{2} \\
\vdots \\
s_{1} \\
s_{2} \\
\vdots
\end{array}\right)=\left(\begin{array}{c}
t_{1} \\
\vdots \\
t_{n} \\
v_{11} \\
\vdots \\
v_{1 n} \\
v_{21} \\
\vdots \\
v_{2 n} \\
\vdots \\
s_{1} \\
s_{2} \\
\vdots
\end{array}\right) \in \mathfrak{R}^{l}
$$

It should be pointed out that a necessary condition for a correct quantification of interference errors is, that the interfering species are represented within the state vector as full profiles with a sufficient number of grid elements $n$. This number must be chosen large enough so that the true profile variations can be properly modeled by the forward model. The reason for this will be detailed in the next section.

\subsection{Quantification of interference errors}

2.2.1 Case I: quantification for fine-grid retrieval of interfering species

The formalism in this section yields reasonable results only if a fine retrieval grid is implemented for the interfering species. We re-arrange and simplify Eq. (A1) to the following form

$\hat{\boldsymbol{x}}-\boldsymbol{x}_{a}=\mathbf{A}\left(\boldsymbol{x}-\boldsymbol{x}_{\alpha}\right)+\boldsymbol{\varepsilon}_{x}$

where $\boldsymbol{\varepsilon}_{x}$ comprises the error terms $2-4$ in Eq. (A1), i.e., all errors in the measurement and the forward model (parameters). $\mathbf{A}$ is the averaging kernel matrix which can be calculated analytically from the following relation (Steck, 2002)

$\mathbf{A}=\left(\mathbf{K}_{x}^{T} \mathbf{S}_{\varepsilon}^{-1} \mathbf{K}_{x}+\mathbf{R}\right)^{-1} \mathbf{K}_{x}^{T} \mathbf{S}_{\varepsilon}^{-1} \mathbf{K}_{x}$,

where $\mathbf{K}_{x}$ is the Jacobian of $\mathbf{F}$ with respect to $\boldsymbol{x}, \mathbf{S}_{\varepsilon}$ is the error covariance of the measurement, and $\mathbf{R}$ is the regularization matrix (see Appendix B, for details).

Note that in Rodgers $(1990,2000) \boldsymbol{x}$ is only the vector of the target species, i.e., what we designated by $t$ above. In this paper, however, we define $\boldsymbol{x}$ as the full state vector comprising vectors for the target species and vectors for all interfering species and additional vector-type quantities retrieved, as well as the retrieved auxiliary scalar parameters, see Eq. (1). In consequence, $\mathbf{A}$ denotes in our case a generalized averaging kernel matrix which includes in addition rows and columns describing the interference of the retrieval of the target profile with the retrieval of all further parame- ters: inserting Eq. (1) into Eq. (2) yields

$$
\begin{aligned}
& \hat{\boldsymbol{x}}-\boldsymbol{x}_{a}=\left(\begin{array}{c}
\hat{\boldsymbol{t}}-\boldsymbol{t}_{a} \\
\hat{\boldsymbol{v}}_{1}-\boldsymbol{v}_{1 a} \\
\hat{\boldsymbol{v}}_{2}-\boldsymbol{v}_{2 a} \\
\vdots \\
\hat{s}_{1}-s_{1 a} \\
\hat{s}_{2}-s_{2 a} \\
\vdots
\end{array}\right) \\
& =\left(\begin{array}{ccccccc}
\mathbf{A}_{t t} & \mathbf{A}_{t v 1} & \mathbf{A}_{t v 2} & \cdots & \boldsymbol{a}_{t s 1}^{T} & \boldsymbol{a}_{t s 2}^{T} & \cdots \\
\mathbf{A}_{v 1 t} & \mathbf{A}_{v 1 v 1} & \mathbf{A}_{v 1 v 2} & \cdots & \boldsymbol{a}_{v 1 s 1}^{T} & \boldsymbol{a}_{v 1 s 2}^{T} & \ddots \\
\mathbf{A}_{v 2 t} & \mathbf{A}_{v 2 v 1} & \mathbf{A}_{v 2 v 2} & \cdots & \boldsymbol{a}_{v 2 s 1}^{T} & \boldsymbol{a}_{v 2 s 2}^{T} & \ddots \\
\vdots & \ddots & \ddots & \ddots & \ddots & \ddots & \ddots \\
\boldsymbol{a}_{s 1 t} & \boldsymbol{a}_{s 1 v 1} & \boldsymbol{a}_{s 1 v 2} & \ddots & a_{s 1 s 1} & a_{s 1 s 2} & \ddots \\
\boldsymbol{a}_{s 2 t} & \boldsymbol{a}_{s 2 v 1} & \boldsymbol{a}_{s 2 v 2} & \ddots & a_{s 2 s 1} & a_{s 2 s 2} & \ddots \\
\vdots & \ddots & \ddots & \ddots & \ddots & \ddots & \ddots
\end{array}\right) \times \\
& \left(\begin{array}{c}
\boldsymbol{t}-\boldsymbol{t}_{a} \\
\boldsymbol{v}_{1}-\boldsymbol{v}_{1 a} \\
\boldsymbol{v}_{2}-\boldsymbol{v}_{2 a} \\
\vdots \\
s_{1}-s_{1 a} \\
s_{2}-s_{2 a} \\
\vdots
\end{array}\right)+\left(\begin{array}{c}
\boldsymbol{\varepsilon}_{t} \\
\boldsymbol{\varepsilon}_{v 1} \\
\boldsymbol{\varepsilon}_{v 2} \\
\vdots \\
\varepsilon_{s 1} \\
\varepsilon_{s 2} \\
\vdots
\end{array}\right) .
\end{aligned}
$$

Our generalized averaging kernel matrix A comprises submatrices $\mathbf{A}_{i j}$, (column) vectors $\boldsymbol{a}_{i j}$, row vectors $\boldsymbol{a}_{j i}^{T}$, as well as scalars $a_{i i}$. Note that $\mathbf{A}_{t t}$ is what is usually called the "averaging kernel matrix" describing the smoothing of the retrieved target profile (Rodgers, 1990, 2000). Furthermore, if the retrieved auxiliary scalar parameters $s_{1}, s_{2}, \ldots$ describe true physical scalar-type quantities (i.e., they are not scalar approximations to a vector-type physical quantity), and they are not correlated, then the retrieval of these scalars can and should be performed without any regularization. In this case Eq. (4) simplifies to

$$
\hat{\boldsymbol{x}}-\boldsymbol{x}_{a}=\left(\begin{array}{c}
\hat{\boldsymbol{t}}-\boldsymbol{t}_{a} \\
\hat{\boldsymbol{v}}_{1}-\boldsymbol{v}_{1 a} \\
\hat{\boldsymbol{v}}_{2}-\boldsymbol{v}_{2 a} \\
\vdots \\
\hat{s}_{1}-s_{1 a} \\
\hat{s}_{2}-s_{2 a} \\
\vdots
\end{array}\right)
$$




$$
\begin{gathered}
=\left(\begin{array}{ccccccc}
\mathbf{A}_{t t} & \mathbf{A}_{t v 1} & \mathbf{A}_{t v 2} & \cdots & 0 & 0 & \cdots \\
\mathbf{A}_{v 1 t} & \mathbf{A}_{v 1 v 1} & \mathbf{A}_{v 1 v 2} & \cdots & 0 & 0 & \ddots \\
\mathbf{A}_{v 2 t} & \mathbf{A}_{v 2 v 1} & \mathbf{A}_{v 2 v 2} & \cdots & 0 & 0 & \ddots \\
\vdots & \ddots & \ddots & \ddots & \ddots & \ddots & \ddots \\
0 & 0 & 0 & \ddots & 1 & 0 & \ddots \\
0 & 0 & 0 & \ddots & 0 & 1 & \ddots \\
\vdots & \ddots & \ddots & \ddots & \ddots & \ddots & \ddots
\end{array}\right) \times \\
\left(\begin{array}{c}
\boldsymbol{t}-\boldsymbol{t}_{a} \\
\boldsymbol{v}_{1}-\boldsymbol{v}_{1 a} \\
\boldsymbol{v}_{2}-\boldsymbol{v}_{2 a} \\
\vdots \\
s_{1}-s_{1 a} \\
s_{2}-s_{2 a} \\
\vdots
\end{array}\right)+\left(\begin{array}{c}
\boldsymbol{\varepsilon}_{t} \\
\boldsymbol{\varepsilon}_{v 1} \\
\boldsymbol{\varepsilon}_{v 2} \\
\vdots \\
\varepsilon_{s 1} \\
\varepsilon_{s 2} \\
\vdots
\end{array}\right)
\end{gathered}
$$

We then obtain the following relation between $\hat{\boldsymbol{t}}, \boldsymbol{t}_{a}$, and $\boldsymbol{t}$

$\hat{\boldsymbol{t}}-\boldsymbol{t}_{a}=\mathbf{A}_{t t}\left(\boldsymbol{t}-\boldsymbol{t}_{\alpha}\right)+\mathbf{A}_{t v 1}\left(\boldsymbol{v}_{1}-\boldsymbol{v}_{1 a}\right)+\mathbf{A}_{t v 2}\left(\boldsymbol{v}_{2}-\boldsymbol{v}_{2 a}\right)+\ldots+\boldsymbol{\varepsilon}_{t},(6)$

which can be rearranged

$$
\begin{aligned}
\hat{\boldsymbol{t}}-\boldsymbol{t}= & \left(\mathbf{A}_{t t}-\mathbf{I}\right)\left(\boldsymbol{t}-\boldsymbol{t}_{\alpha}\right) \quad \ldots \text { smoothing error } \\
& +\mathbf{A}_{t v 1}\left(\boldsymbol{v}_{1}-\boldsymbol{v}_{1 a}\right)+\mathbf{A}_{t v 2}\left(\boldsymbol{v}_{2}-\boldsymbol{v}_{2 a}\right) \ldots \text { interference error } \\
& +\ldots \\
& +\boldsymbol{\varepsilon}_{t}
\end{aligned}
$$

The first term is what has been defined by Rodgers (1990, $2000)$ as the "smoothing error" since it describes the differences between the retrieved profile $\hat{\boldsymbol{t}}$ and the true profile $\boldsymbol{t}$; these differences are due to the finite vertical resolution of the remote sounding system, or, in other words, due the fact that $\mathbf{A}_{t t}$ of real remote sounders deviates from the ideal unit matrix $\mathbf{I}$. The further terms are what we will refer to hereafter as "interference errors". They are caused by interference between the retrieval of the target profile $t$ and the retrieval of the interfering species with profiles $\boldsymbol{v}_{1}, \boldsymbol{v}_{2}, \ldots$ (including retrieved auxiliary quantities, e.g., temperature). We will call $\mathbf{A}_{t v 1}, \mathbf{A}_{t v 2}, \ldots$ "interference kernel matrices". In analogy to the term "averaging kernels" we hereafter use the term "interference kernels" for the rows of these interference kernel matrices. In consequence, an interference kernel for a certain nominal layer altitude monitors the response of the target profile retrieval at this layer altitude to a unit perturbation of the true profile of the interfering species at all the different layer altitudes of the retrieval grid (in arbitrary units of the state vector quantity, e.g., scaling factors of VMR-layer averages).

The statistics of the smoothing error is described by the error covariance

$\mathbf{S}_{t t}=\left(\mathbf{A}_{t t}-\mathbf{I}\right) \mathbf{S}_{t}\left(\mathbf{A}_{t t}-\mathbf{I}\right)^{T}$, where $\mathbf{S}_{t}$ is a best estimate of the true a priori covariance of the target profiles $t$.

The statistics of the interference errors are described by the error covariance matrices

$$
\begin{gathered}
\mathbf{S}_{t v 1}=\mathbf{A}_{t v 1} \mathbf{S}_{v 1} \mathbf{A}_{t v 1}^{T} \\
\mathbf{S}_{t v 2}=\mathbf{A}_{t v 2} \mathbf{S}_{v 2} \mathbf{A}_{t v 2}^{T} \\
\vdots
\end{gathered}
$$

where $\mathbf{S}_{v 1}, \mathbf{S}_{v 2}, \ldots$ are best estimates of the true a priori covariances of the profiles $\boldsymbol{v}_{1}, \boldsymbol{v}_{2}, \ldots$ of the interfering species.

\subsubsection{Case II: quantification in the case of coarse-grid re- trieval of interfering species}

Many operational algorithms are using coarse-grid retrievals for the interfering species due to computation power limitations. For instance, in ground-based FTIR spectrometry, it is still a widely used practice to retrieve the interfering species only via one VMR-profile scaling factor per species.

However, in the case of a coarse-grid retrieval of the interfering species there is no appropriate interface to link the true atmospheric (high-resolution) profile covariance of the interfering species into the interference error analysis. In consequence, direct application of Eqs. $(3,4,9)$ to the coarse grid leads to erroneous results, i.e., significant underestimations of the true interference errors. This may even lead - wrongly - to zero interference errors: e.g., in the case of a simple unconstrained VMR-profile scaling retrieval of the interfering species, the (erroneous) application of Eqs. $(3,4,9)$ on this coarse (1-layer) grid would mean to use only one additional scalar entry for the profile of an interfering species to construct the generalized state vector, instead of $n$ additional entries describing its profile. In this case the interference kernels computed via Eqs. (3) and (4) would no longer be a $n \times n$-matrix, but a $n$-dimensional row vector with value $\boldsymbol{a}_{t v 1}=(00 \ldots 0)$. Consequently, the interference error calculated according to Eq. (9) would be (scalar) zero. Of course this is not true. The reason for is the implicitly made erroneous assumption that the true atmospheric variability of the interfering species is only of profile-scaling type, which means there would be no changes in profile shape (which is wrong, of course). The algorithmic effect leading to the error is then the well known fact, that an unconstrained VMRprofile scaling retrieval is always able to perfectly retrieve any profile change corresponding to a constant difference relative to the a priori profile. In other words, the retrieval of the interfering species would not lead to any residual under this assumption and, in consequence, there would be no interference effect on the target species. In a similar way, direct application of Eqs. $(3,4,9)$ to all other coarse-grid retrievals of interfering species (dividing their vertical profiles into 2 , $3, \ldots$ layers) will always lead to a serious underestimate of the true interference errors. 
In order to overcome this difficulty, we have to implement a sufficiently fine retrieval grid for the interfering species and emulate their coarse-grid retrieval (namely that of the operational algorithm to be characterized). This can be achieved by using an appropriate soft constraint $\mathbf{R}$ (for definition, see Appendix B, Eq. B1) for the interfering species.

An unconstrained retrieval of the interfering species on a (coarse) 1-layer grid (e.g., VMR-profile scaling) can be emulated on a $n$-layer fine grid using the Tikhonov-type first order regularization matrix (see Appendix B) for the interfering species

$$
\mathbf{R}_{\text {one block }}=\alpha \times\left(\begin{array}{ccccc}
1 & -1 & 0 & \cdots & 0 \\
-1 & 2 & \ddots & \ddots & \vdots \\
0 & \ddots & \ddots & \ddots & 0 \\
\vdots & \ddots & \ddots & 2 & -1 \\
0 & \cdots & 0 & -1 & 1
\end{array}\right) \in \Re^{n \times n}
$$

with a very high regularization strength, i.e., $\alpha \rightarrow \infty$.

The emulation of other $(2,3, \ldots$-layer $)$ coarse-grid retrievals of the interfering species on a $n$-layer fine grid is straightforward if we use the following multi-blockTikhonov regularization

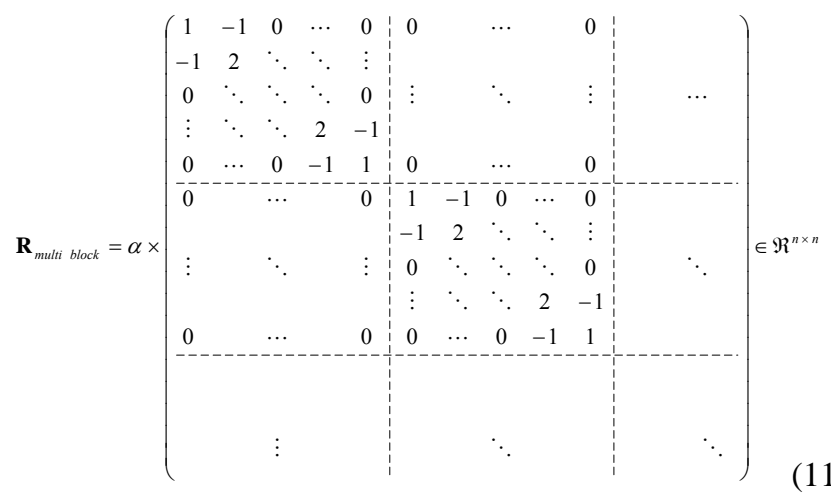

for the interfering species, again with $\alpha \rightarrow \infty$ (we assume here that the fine grid is designed so that it contains the altitude borders of the coarse grid).

After having emulated the coarse-grid retrieval of the interfering species on a fine grid, the quantification of interference errors can be performed by straightforward application of Eqs. $(3,4,9)$ on the fine grid.

2.2.3 Case III: quantification in the case of unretrieved interfering species

In satellite infrared remote sensing, interfering species are sometimes not retrieved due to computation power limitations. Interference errors originate in this case from a different effect. They do not arise from the regularization of their retrieval (because they are not retrieved), but from insufficient knowledge of their abundances in the forward model, which again leads to spectral residuals that hinder the retrieval of the target species. Case III, therefore, is the only subgroup of interference errors that could be quantified by classical error analysis, i.e, the concept of model parameter errors, as defined in Rodgers (2000, Eq. 3.16, second term). Implementation of this approach is somewhat laborious since the climatological covariance of the interfering species has to be transferred to measurement space (via Jacobian $\mathbf{K}_{b}$, which has to be calculated, see Appendix A) and then mapped back to state space to quantify the impact upon the target species retrieval. This has been performed before (e.g., von Clarmann and Echle, 1998; Echle et al., 2000; Dudhia et al., 2002).

We suggest here a simple alternative way to characterize the errors from unretrieved interfering species directly in state space: in case of algorithms where the interfering species are readily implemented on a fine grid within the state vector, Eqs. $(3,4,9)$ can be directly applied (using $\mathbf{K}_{x}$ which is readily available) by formally retrieving the interfering species, but using a simple "dead regularization" of the form

$\mathbf{R}_{\text {dead }}=\beta \times\left(\begin{array}{cccc}1 & 0 & \cdots & 0 \\ 0 & 1 & \ddots & \vdots \\ \vdots & \ddots & \ddots & 0 \\ 0 & \cdots & 0 & 1\end{array}\right) \in \Re^{n \times n}$

with $\beta \rightarrow \infty$. This emulates a non-retrieval of the interfering species while preserving their fine-grid entry to the state vector, which allows the correct application of Eqs. $(3,4,9)$. This alternative approach may be used, e.g., for a quick test of the effect of non-retrieving versus retrieving a certain interfering species.

2.3 Treatment of non-linearity for quantification of interference errors and smoothing errors

To solve the inverse problem and to analyze smoothing and interference errors we use the following linearization of the forward model

$$
\begin{aligned}
\boldsymbol{y} & =\mathbf{F}\left(\boldsymbol{x}_{0}, \boldsymbol{b}_{0}\right)+\left.\frac{\partial \mathbf{F}}{\partial \boldsymbol{x}}\right|_{x_{0}, b_{0}}\left(\boldsymbol{x}-\boldsymbol{x}_{0}\right)+\boldsymbol{\varepsilon}_{y} \\
& =\left.\mathbf{K}\right|_{x_{0}, b_{0}}\left(\boldsymbol{x}-\boldsymbol{x}_{0}\right)+\boldsymbol{\varepsilon}_{y}
\end{aligned}
$$

around a linearization point $\boldsymbol{x}_{0}, \boldsymbol{b}_{0}$.

Conventionally, the error analysis is performed via linear approximation of the forward model using a single linearization point. The result would be one "typical" smoothing error covariance and one interference error covariance.

However, the forward model is often not sufficiently linear, i.e., it does not allow for an appropriate description of the interference errors and smoothing errors for the full ensemble of all possible values of parameters $\boldsymbol{x}$ and $\boldsymbol{b}$ using only one linearization. Any sub-states of $\boldsymbol{x}$ or any $\boldsymbol{b}$ parameters that cause strong changes between different spectra are responsible for this kind of situation. In this case, an appropriate description can be found by using a statistical approach based 
on an ensemble of $i$ linearizations with linearization points $\left(\hat{\boldsymbol{x}}_{i}, \boldsymbol{b}_{i}\right)$, i.e., the $i$ retrieved states $\hat{\boldsymbol{x}}_{i}$ and the corresponding input forward model parameters $\boldsymbol{b}_{i}$ that have been used for each retrieval. Doing so, one will typically find a strong dependency of the resulting interference errors and smoothing errors on the state of the highly variable atmospheric water vapor (either playing the role of an interfering species or target species), as well as on the solar zenith angle (SZA) in case of solar absorption spectrometry, or the tangent altitude in case of satellite limb sounding.

\subsection{Minimization of interference errors}

In the following we discuss influencing factors that should be considered for minimization of interference errors. All factors also affect smoothing errors, which will therefore be discussed in parallel. The points made will be illustrated for a real sounding in Sect. 4.

\subsubsection{Impact of the constraint applied to interfering species}

Reducing the (hard or soft) constraint applied to interfering species has two effects. i) interference errors are reduced, and ii) the smoothing error is increased. We explain this using the example of a totally unconstrained retrieval as follows.

i) Interference errors are completely eliminated by using a totally unconstrained profile retrieval of the interfering species on a fine grid. This is because their unconstrained retrieval yields a perfect fit (neglecting model parameter errors and forward model errors), i.e., it does not cause any residuals that would affect the retrieval of the target species. The unconstrained retrieval of the interfering species may lead to oscillating profiles for the interfering species (because the model parameter errors, forward model errors, and retrieval noise errors for the retrieved profile of the interfering species increase), but this does not have an effect on the retrieval of the target profile.

ii) An unconstrained fine-grid profile retrieval of the interfering species leads to an increased smoothing error for the target species in comparison with any constrained case. This is because an unconstrained retrieval of the interfering species increases the solution space for the retrieved profiles of the interfering species, which corresponds to a certain kind of deweighting in measurement space around the signature of the interfering species and around the overlapping features of the target species. This leads to a reduced information content for retrieval of the target species, i.e., an increased smoothing error.

Therefore, the following hierarchy holds for the magnitude of interference errors (on average over a test ensemble of spectra) for different typical algorithmic constraints imposed on the interfering species: interference errors when the interfering species are not retrieved (infinitely strong constraint) $>$ interference errors when the interfering species are retrieved via VMR-profile scaling (hard constraint on profile shape) $>$ interference errors when the interfering species are retrieved on a fine grid with some soft constraint $>$ interference errors when the interfering species are retrieved on a fine grid without any soft constraint.

For smoothing errors, the reverse hierarchy holds (on average): smoothing errors when the interfering species are not retrieved $<$ smoothing errors when the interfering species are retrieved via VMR-profile scaling $<$ smoothing errors when the interfering species are retrieved on a fine grid with some soft constraint $<$ smoothing errors when the interfering species are retrieved on a fine grid without any soft constraint.

\subsubsection{Optimum strategy for retrieval of interfering species}

The findings of Sect. 2.4.1 imply that in the case of fine-grid profile retrieval of the interfering species, there is a tradeoff between minimizing interference errors and the smoothing error. This is because both errors depend on the regularization strength applied to the retrieval of the interfering species. Therefore, a total minimum of the combined error (from interference and smoothing) can be found. In order to perform this minimization of regularization of the interfering species in a systematic way, we implement a Tikhonov-type firstorder regularization for the interfering species, since this allows the regularization strength to be easily tuned using only one parameter $\alpha$ (see Appendix B for details). For the minimization procedure, we also need to derive scalar error quantities from the error covariances $\mathbf{S}_{t t}$ and $\mathbf{S}_{t v 1}, \mathbf{S}_{t v 2} ., \ldots$ There are several ways to do this, depending on the target of error minimization. We suggest the use of, e.g., "mean errors"

$$
\begin{aligned}
\bar{\sigma}_{t t}:= & \sqrt{\sum_{i=1}^{n}\left(\mathbf{S}_{t t}\right)_{i i} / n}, \ldots \text { mean smoothing error } \\
\bar{\sigma}_{t v 1}:= & \sqrt{\sum_{i=1}^{n}\left(\mathbf{S}_{t v 1}\right)_{i i} / n}, \\
& \ldots \text { mean interference error from species } \boldsymbol{v}_{1} \\
& \vdots
\end{aligned}
$$

Then, an optimum $\alpha_{v 1}$ for the retrieval of the interfering species $\boldsymbol{v}_{1}$ can be found by searching for the minimum of the combined error

$\bar{\sigma}_{\mathrm{comb}}\left(\alpha_{v 1}\right)=\operatorname{sqrt}\left(\bar{\sigma}_{t t}^{2}\left(\alpha_{v 1}\right)+\bar{\sigma}_{t v 1}^{2}\left(\alpha_{v 1}\right)\right)$.

For the other interfering species $\boldsymbol{v}_{2}, \ldots$ the optimum $\alpha_{v 2}, \ldots$ can be found by analogy. See Sect. 4.2, for an illustration of this optimization procedure using a real sounding.

It should be noted that we suggest a Tikhonov-type regularization for the retrieval of the interfering species here, because of the practical advantage that the regularization strength can be tuned by a single scalar parameter, $\alpha$. Details of the retrieved interfering profiles are not of interest. They will casually suffer from oscillations for the optimum 
$\alpha$, which may be found to be a very small number. This is because the optimization is at the cost of increasing model parameter errors, forward model errors, and retrieval noise errors for the profiles of the interfering species. But this does not matter, i.e., it does not impair target species retrieval. Note, that we have to take leave here of the idea of optimal estimation for retrieval of the interfering species: application of optimal estimation in the strict sense for the retrieval of the interfering species, i.e., the use of a climatological constraint $\mathbf{R}=\mathbf{S}_{R}^{-1}$ (see Appendix B) would in general lead to larger interference errors as compared with our optimized Tikhonov approach and never to smaller interference errors. This is because our approach uses a systematically minimized constraint $\alpha \mathbf{L}^{T} \mathbf{L}$ for the interfering species, which will always be softer than any fixed climatological constraint $\mathbf{S}_{R}^{-1}$, if the optimization requires this - leading to smaller residuals from the interfering species. This being said, the target species can still be retrieved via optimal estimation, if preferred.

We want to make one additional note about model parameter errors, forward model errors, and retrieval noise errors (see Eq. A1), which we have intentionally not treated in our paper. One might wonder whether errors belonging to these three classes might increase or decrease due to application of our optimum strategy for retrieval of the interfering species. The answer is that application of our optimum strategy leads always to a reduction of these further errors. This can be seen from analogy to the finding by Steck (2002, see Fig. 4 therein): as a result of a change in target species regularization strength to higher (lower) values, the smoothing error is also shifted to higher (lower) values, and both forward model (parameter) errors and retrieval noise errors are shifted to lower (higher) values. This finding for a varied target species regularization can be transferred to our case of varied strength of interfering species regularization since both are linked together (in the opposite direction). As explained in Sect. 2.4.1, any decrease (increase) in the regularization strength of the interfering species increases (decreases) the solution space for the interfering species, which in measurement space has the side-effect of weighting the target species retrieval to a lower (higher) degree. This means that forward model (parameter) errors and retrievals noise are reduced (increased).

\subsubsection{Impact of microwindow selection}

The question is whether the interference errors as well as the overall (smoothing and interference) error are decreased or increased by widening microwindows. The answer is nontrivial, i.e., the sign of the net effect depends on the type of constraint applied to the interfering species, in detail as follows.

\section{Impact in case interfering species are not retrieved}

If the interfering species are not retrieved, the interference error will dramatically increase for a widened microwindow which is additionally including significant signatures of the interfering species. At the same time, a widened microwindow increases information content for the target species slightly, i.e., the smoothing error is slightly decreased. The net effect will be a significant increase of the combined error (comprising smoothing error and interference errors). Therefore, if the interfering species cannot be retrieved due to computation power limitations (e.g., this is the case in some satellite retrievals), minimization of the interference effect can be taken care of by systematic microwindow selection, targeting at minimal inclusion of strong signatures of the interfering species, while preserving the main features of the target species as well as possible (e.g., von Clarmann and Echle, 1998; Echle et al., 2000; Dudhia et al. 2002). Impact in case the optimum strategy for retrieval of interfer-
ing species is applied

In case the optimum strategy (proposed in Sect. 2.4.2) is implemented for retrieval of the interfering species, a widening of microwindows is uncritical, i.e., it does not increase the combined error (comprising smoothing error and interference error), and it leads to a slightly smaller combined error instead. Note that this is just the opposite of what holds true for the case of unretrieved interfering species (see preceding section).

The reason for this is that in case of a fine-grid profile retrieval of the interfering species with minimized regularization strength, the interference error has been reduced below the smoothing error (optimum strategy proposed in Sect. 2.4.2). Therefore, widening microwindows now has the main effect of increasing the information content for the target species, and a new optimization will again reduce the interference error below the smoothing error. The net effect will be that the combined error (from interference and smoothing) will be (slightly) reduced by widening microwindows (at the cost of increased computation time). In other words, the details of microwindow selection become uncritical for a setup using fine-grid profile retrieval of the interfering species with minimized regularization strength (optimum strategy proposed in Sect. 2.4.2). This is a crucial point of our paper. It will be demonstrated for a real sounding in Sect. 4.3.2.

\section{Interference errors in the standard retrieval of $\mathrm{CO}$ profiles from solar FTIR spectrometry}

\subsection{Historical development of CO solar spectrometry}

CO profiling via solar FTIR spectrometry was developed and refined in a series of papers (Pougatchev and Rinsland, 1995; 
Zhao et al., 1997; Rinsland et al., 1998, 2000, 2002) finally utilizing a set of lines (R3, P7, P10) of the 1-0 band, and optimal estimation (Rodgers, 1976). A detailed error analysis of the $\mathrm{CO}$ retrieval was presented by Rodgers and Connor (2003) according to the Rodgers (1990, 2000) formalism. A variety of applications have been reported, e.g., for investigating the impact of biomass burning on the global $\mathrm{CO}$ distribution (e.g., Jones et al., 2001; Yurganov et al., 2004, 2005; Velazco et al., 2005; Paton-Walsh et al., 2005), as well as for satellite validation (e.g., Pougatchev et al., 1998; Rodgers and Connor, 2003; Sussmann and Buchwitz, 2005).

\subsection{Zugspitze FTIR CO measurements}

At the NDACC Primary Station Zugspitze $\left(47.42^{\circ} \mathrm{N}\right.$, $10.98^{\circ}$ E, $2964 \mathrm{~m}$ a.s.l.), Germany, a Bruker $120 \mathrm{HR}$ solar FTIR instrument was setup at the beginning of 1995 (Sussmann and Schäfer, 1997). Since then it has been operated all year round with typically 120 measurement days per year, and is part of the Permanent Ground-Truthing Facility Zugspitze/Garmisch (Sussmann and Buchwitz, 2005; Sussmann et al., 2005a, b).

\subsubsection{Test ensemble of Zugspitze spectra}

A typical Zugspitze spectrum is the average of 6 scans with an optical path difference of $250 \mathrm{~cm}^{-1}$, recorded within $14 \mathrm{~min}$. For this paper we randomly selected a test ensemble of 156 spectra taken after 1994. The average signal-torms-noise ratio of the spectra of this ensemble is $377: 1$. A significant problem in the forward model between 2157.77$2157.92 \mathrm{~cm}^{-1}$ was found in the final residuals of the spectral fits (measured minus calculated). The next section explains the reason for this.

\subsubsection{Contribution plot and forward model characteristics}

In the following we will characterize the standard $\mathrm{CO}$ retrieval using the microwindow set given by Rinsland et al. (2000) (2057.78-2057.91 cm $\mathrm{cm}^{-1}, 2069.61-2069.71 \mathrm{~cm}^{-1}$, and $\left.2157.30-2159.15 \mathrm{~cm}^{-1}\right)$. Figure 1 shows a contribution plot (colored lines). It has been calculated using the average SZA and the average of the retrieved states $\hat{\boldsymbol{x}}_{i}$ of our test ensemble. Note that there are four terrestrial interfering species, i.e., $\mathrm{O}_{3}, \mathrm{H}_{2} \mathrm{O}, \mathrm{N}_{2} \mathrm{O}$, and $\mathrm{CO}_{2}$. Furthermore, it can be seen that the residual problem found between 2157.77$2157.92 \mathrm{~cm}^{-1}$ (as mentioned in Sect. 3.2.1) is due to a solar $\mathrm{CO}$ line that has not been adequately modeled.

For the forward simulations we used the HITRAN 2004 spectroscopic line parameter compilation (Rothmann et al., 2005). For the pressure-temperature profile information used in the forward model we utilized the daily Munich radio sonde launched at 12:00 universal time about $80 \mathrm{~km}$ north of the Zugspitze.
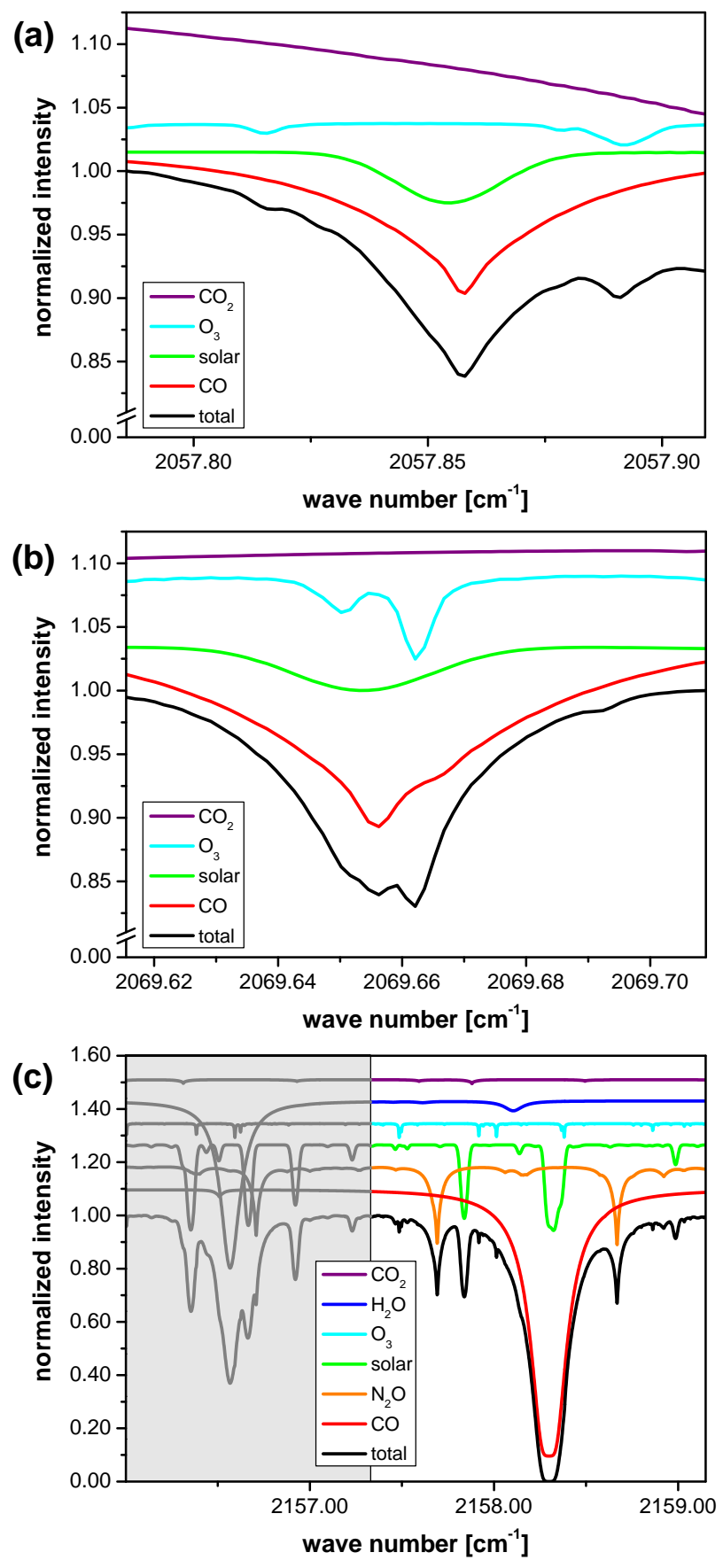

Fig. 1. Forward calculation of the three micro-windows (a-c) of the solar infrared absorption spectrum used for $\mathrm{CO}$ profile retrievals. The contributions of the different absorbing species are separated. Note, that the grey-shaded spectral area in (c) is not used for the standard retrieval. It is only used for the sensitivity study with the widened microwindow in Sect. 5.1.

\subsection{The standard CO retrieval settings}

We use the SFIT2 (ver. 3.90) software with an equidistant $1-\mathrm{km}$-layer retrieval grid for the target species and follow 

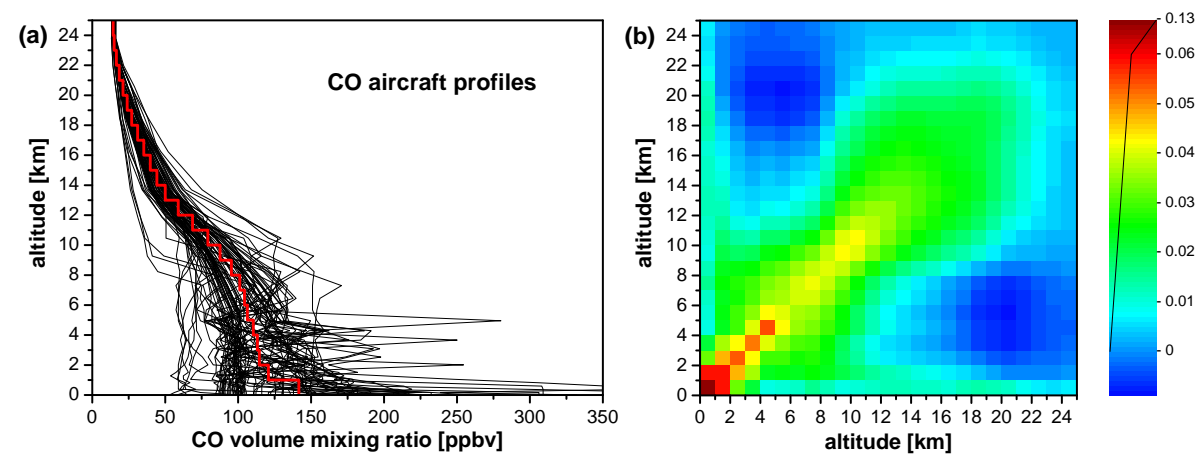

Fig. 2. Climatological ensemble of CO aircraft profiles used to construct the Zugspitze CO a priori profile (a) and the CO a priori covariance (b). Note, that the units of the state vector are scaling factors for the VMR-layer averages of the a priori profile given on a $1 \mathrm{~km}$ grid.

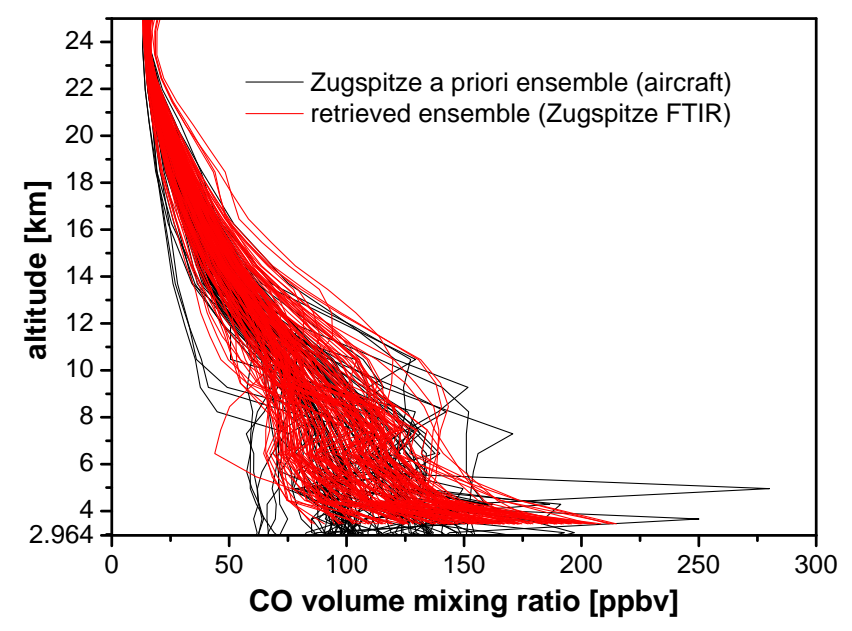

Fig. 3. CO profiles retrieved via optimal estimation from a test ensemble of 156 Zugspitze solar FTIR spectra plotted together with the climatological aircraft profile ensemble used to construct the a priori information (see also Fig. 2). The CO standard retrieval including a VMR-profile scaling retrieval for all interfering species has been used with the Rinsland et al. (2000) microwindows.

the RRC retrieval approach as described by Rinsland et al. (2000) and Rodgers and Connor (2003), with the modifications that follow.

\subsubsection{CO a priori profile and full covariance for mid lati-} tudes

To extend the RRC approach, we used a full CO a priori covariance matrix for the Zugspitze retrievals. This matrix was constructed from an ensemble of measured high-resolution profiles. Earlier RRC had used a simple empirical a priori covariance matrix for $\mathrm{CO}$ comprising diagonal elements only, with the standard deviations (stdv) for all layers either varied smoothly from $40 \%$ below $30 \mathrm{~km}$ to $20 \%$ above $40 \mathrm{~km}$ (Rinsland et al., 2000), or all set to $100 \%$ (Rodgers and Connor, 2003). While these RRC retrieval settings were an empirical approach to stabilize the retrieval without too much influence from a priori information, the Zugspitze approach employs a strict application of the optimal estimation concept to the retrieval of CO profiles from solar FTIR.

The Zugspitze a priori profile $\boldsymbol{t}_{a}$ and a priori covariance matrix $\mathbf{S}_{t}=\mathbf{S}_{\mathrm{CO}}$ for optimal estimation of $\mathrm{CO}$ was constructed from an ensemble of globally distributed aircraft $\mathrm{CO}$ measurements supplemented above the aircraft altitudes by a set of modeled profiles used for the operational MOPITT retrieval (Deeter et al., 2003). We selected a subset comprising all profiles within a full $47^{\circ} \mathrm{N} \pm 16^{\circ}$ latitudinal band (Fig. 2a). Figure 2a also shows the mean profile of this ensemble which is used as an a priori profile $\boldsymbol{t}_{a}$. The resulting a priori covariance matrix $\mathbf{S}_{t}=\mathbf{S}_{\mathrm{CO}}$ was calculated from the statistics of the ensemble (Fig. 2b).

\subsubsection{Error covariance $\mathbf{S}_{\varepsilon}$ and deweighting}

The measurement error covariance matrix $\mathbf{S}_{\varepsilon}$ was assumed to be diagonal. For the uncertainties of all spectral channels the average signal-to-noise ratio of the Zugspitze test ensemble was used (377:1). To prevent the forward model error around 2157.77-2157.92 $\mathrm{cm}^{-1}$ (see Sects. 3.2.1 and 3.2.2) from being mapped into the retrieval, we performed a total deweighting by assuming a signal-to-noise ratio of 0:1 for this spectral domain within the $\mathbf{S}_{\varepsilon}$ matrix.

\subsubsection{Retrieval of interfering species and auxiliary scalar parameters}

In the standard RRC approach all four interfering species are retrieved via VMR-profile scaling. The following auxiliary scalar parameters were retrieved. There is one independent frequency shift per microwindow (i.e., 3 parameters in total). One additional parameter is needed to fit possible zero line distortions via the saturated R3 line. Three more parameters are used to fit the background slope in each micro-window. There is also one auxiliary parameter to fit a frequency shift to the solar $\mathrm{CO}$ spectrum. 


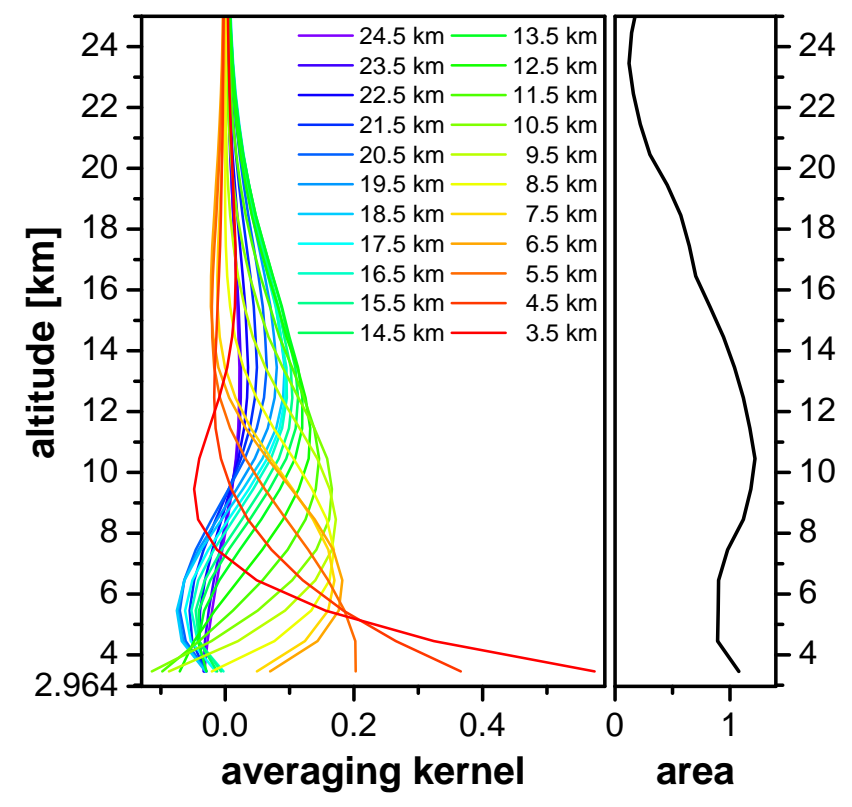

Fig. 4. Averaging kernels (rows of the averaging kernel matrix) for the Zugspitze standard retrieval of CO profiles via optimal estimation. The nominal altitudes of the kernels are given as well as the areas of the kernels as a function of altitude (black curve in the right part). The CO standard retrieval including a VMR-profile scaling retrieval for all interfering species has been used with the Rinsland et al. (2000) microwindows. The kernels plotted are the average of the kernels calculated around all states retrieved from the Zugspitze test ensemble of 156 spectra. Note, that the units of the state vector are scaling factors for the VMR-layer averages of the a priori profile given on a $1 \mathrm{~km}$ grid.

\subsubsection{Retrieved test ensemble}

Figure 3 shows the retrieved CO profiles from an arbitrarily chosen test ensemble of 156 Zugspitze spectra. It can be seen that the overall range of scatter of the retrieved ensemble is consistent with the ensemble of the aircraft profiles (also shown) from which our prior information (covariance and mean profile) was constructed.

\subsection{Quantification of CO smoothing error}

Averaging kernels for the standard $\mathrm{CO}$ profile retrieval (i.e., the rows of $\mathbf{A}_{t t}=\mathbf{A}_{\mathrm{CO}-\mathrm{CO}}$, see Eq. 4) are plotted in Fig. 4. The plotted averaging kernels are averages of the averaging kernels calculated around all the retrieved states of the Zugspitze test ensemble (Fig. 3), i.e., they describe the mean retrieved state. The kernels peak close to their nominal altitude and retain close to unit area up to an altitude of $15 \mathrm{~km}$. The degree of freedom of signal is dofs $=3.3$ on average over our test ensemble.

We calculate the smoothing error covariance $\mathbf{S}_{t t}=\mathbf{S}_{\mathrm{CO}-\mathrm{CO}}$ according to Eq. (8) using the a priori covariance $\mathbf{S}_{t}=\mathbf{S}_{\mathrm{CO}}$ of Fig. 2 b. Figure 5 shows the square roots of the diagonal el-

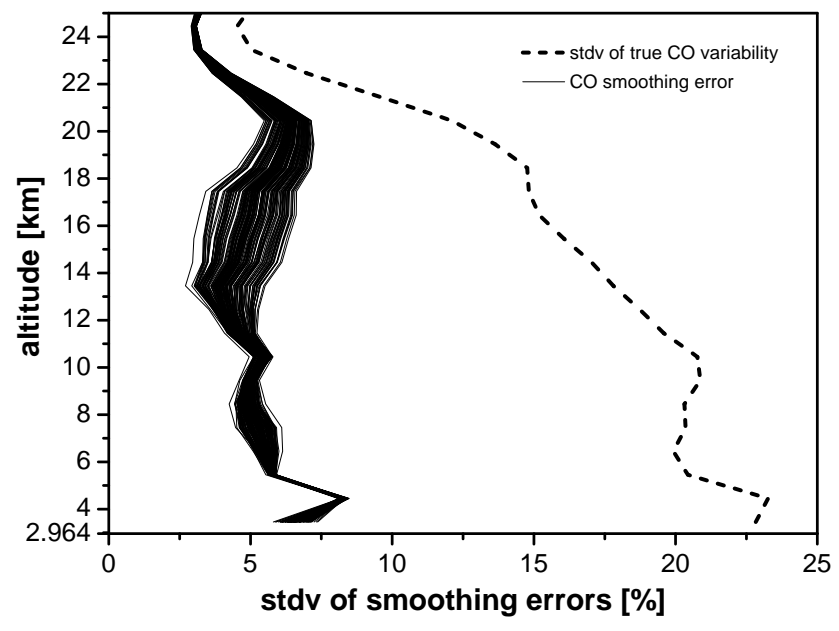

Fig. 5. Profile of the stdv of the true $\mathrm{CO}$ variability (dashed line: square roots of the diagonal elements of the climatological $\mathrm{CO}$ covariance) and ensemble of smoothing error profiles (solid lines: square roots of the diagonal elements of the smoothing error covariance) calculated around all states retrieved from the Zugspitze test ensemble of 156 spectra. The CO standard retrieval including a VMR-profile scaling retrieval for all interfering species has been used with the Rinsland et al. (2000) microwindows.

ements of $\mathbf{S}_{\mathrm{CO}-\mathrm{CO}}$, i.e., error standard deviations as profiles versus altitude. Note that this is not a complete description of the smoothing errors, because they are correlated between different heights. However, it does provide an indication of the retrieval precision. Figure 5 shows the full ensemble of smoothing error profiles calculated around each retrieved state $\left(\hat{\boldsymbol{x}}_{i}, \boldsymbol{b}_{i}\right)$ of the Zugspitze test ensemble. As described in Sect. 2.3, the reason for the spread of the smoothing errors is the non-linearity of the forward model and thus the dependency of the averaging kernels on the state $\left(\boldsymbol{x}_{i}, \boldsymbol{b}_{i}\right)$. We found that the major impact is due to the changing SZA.

Figure 5 also shows the natural $\mathrm{CO}$ variability as a function of altitude which has been calculated as the square root of the diagonal elements of the a priori covariance $\mathbf{S}_{t}=\mathbf{S}_{\mathrm{CO}}$ (Fig. 2b). It can be seen that the magnitude of the smoothing error relative to the natural $\mathrm{CO}$ variability increases with altitude. However, the smoothing error of our retrieval never exceeds the magnitude of the natural $\mathrm{CO}$ variability, as expected for a properly set optimal estimation approach.

\subsection{Quantification of interference errors for the CO stan- dard retrieval}

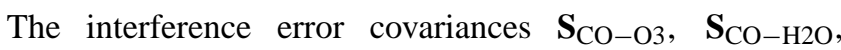

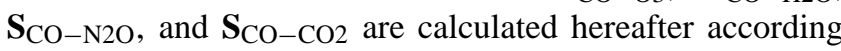
to Eq. (9). As an input to this we first have to calculate the

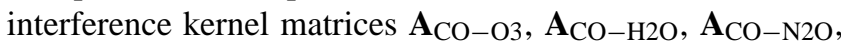
and $\mathbf{A}_{\mathrm{CO}-\mathrm{CO} 2}$ (Sect. 3.5.1). The second set of inputs to Eq. (9) are the a priori covariances for the interfering species $\mathbf{S}_{v 1}=\mathbf{S}_{\mathrm{O} 3}, \mathbf{S}_{v 2}=\mathbf{S}_{\mathrm{H} 2 \mathrm{O}}, \mathbf{S}_{v 3}=\mathbf{S}_{\mathrm{N} 2 \mathrm{O}}$, and $\mathbf{S}_{v 4}=\mathbf{S}_{\mathrm{CO} 2}$, which are 

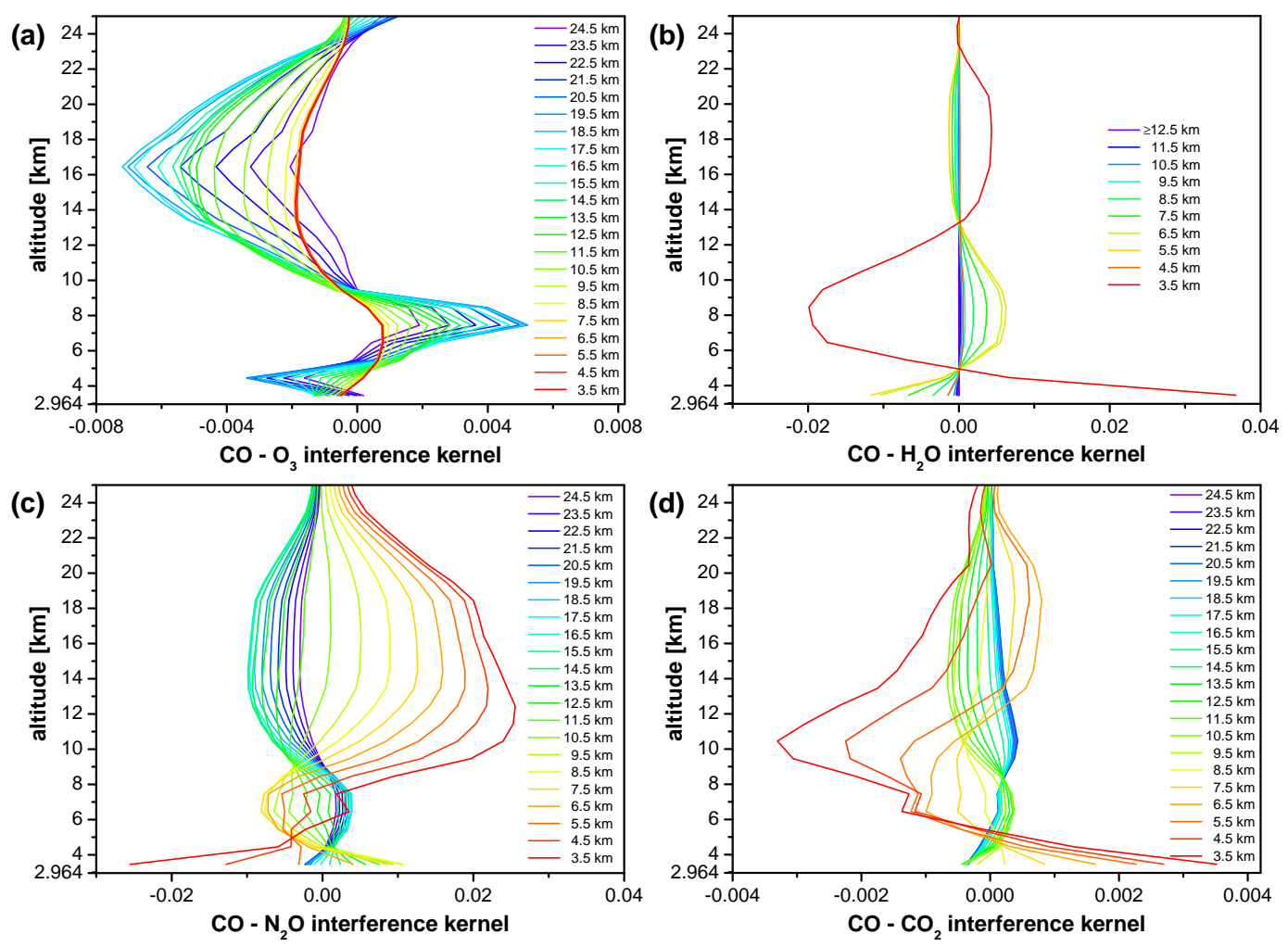

Fig. 6. Interference kernels (rows of the interference kernel matrices) for the interfering species $\mathrm{O}_{3}(\mathbf{a}), \mathrm{H}_{2} \mathrm{O}(\mathbf{b}), \mathrm{N}_{2} \mathrm{O}(\mathbf{c})$, and CO $\mathrm{CO}_{2}$ (d). The kernels are calculated for the CO standard retrieval with the Rinsland et al. (2000) microwindows and a VMR-profile scaling retrieval for all interfering species. The nominal altitudes of the kernels are given. The interference kernels plotted are the average of the kernels calculated around all states retrieved from the Zugspitze test ensemble of 156 spectra. Note, that the units of the state vector are scaling factors for the VMR-layer averages of the a priori profiles given on a $1 \mathrm{~km}$ grid.

presented in Sect. 3.5.2. Then Sect. 3.5.3 shows the resulting interference errors versus altitude and their comparison to the smoothing errors as well as to the natural variability of $\mathrm{CO}$.

\subsubsection{Interference kernels}

The interference kernels for the four interfering species are plotted in Figs. 6a-d. They characterize the standard RRC retrieval which uses a 1-layer coarse-grid retrieval for the four interfering species (VMR-profile scaling). In order to be able to calculate these interference kernels via Eqs. $(3,4)$ we emulated this 1-layer grid on a fine retrieval grid as explained in Sect. 2.2.2. For this purpose we implemented for the interfering species the same equidistant $1-\mathrm{km}$-layer retrieval grid as used for the $\mathrm{CO}$ (target species) retrieval, and applied for their retrieval regularization matrices as given in Eq. (10) with $\alpha=10^{13}$.

\subsubsection{A priori covariances of the interfering species}

To construct the a priori covariance $\mathbf{S}_{v 1}=\mathbf{S}_{\mathrm{O} 3}$ needed to estimate the $\mathrm{CO}-\mathrm{O}_{3}$ interference error we used an ensemble of 1438 ozone sonde (brewer mast) profiles provided by the meteorological observatory Hohenpeissenberg located $30 \mathrm{~km}$ north of the Zugspitze. These soundings are performed 3 times a week, and our ensemble covers the time span January 1995-February 2006. Figure 7a shows the profile ensemble and its mean, and Fig. $7 \mathrm{~b}$ shows the covariance calculated from this ensemble.

To construct the a priori covariance $\mathbf{S}_{v 2}=\mathbf{S}_{\mathrm{H} 2 \mathrm{O}}$ we utilized the data set of the (4 times daily) radio soundings performed during the Garmisch AIRS validation campaign between 19 August-17 November 2002. Garmisch is located horizontally only $6 \mathrm{~km}$ away from the Zugspitze. We used a subset of 66 radio sondes that had been launched coincident to the solar FTIR measurements (i.e., filtering for clear sky conditions). This ensemble of water vapor profiles and the resulting covariance is plotted in Fig. 8.

To estimate the a priori covariance $\mathbf{S}_{v 3}=\mathbf{S}_{\mathrm{N} 2 \mathrm{O}}$ we used an ensemble of 14 aircraft profiles from a number of campaigns performed from 1995-1997 between $20-70^{\circ} \mathrm{N}$ which were provided by the ETHmeg data base (http://www.megdb.ethz. ch/dbaccess.php), see Fig. 9.

The a priori covariance $\mathbf{S}_{v 4}=\mathbf{S}_{\mathrm{CO} 2}$ was constructed from 134 profiles measured during two aircraft campaigns in 

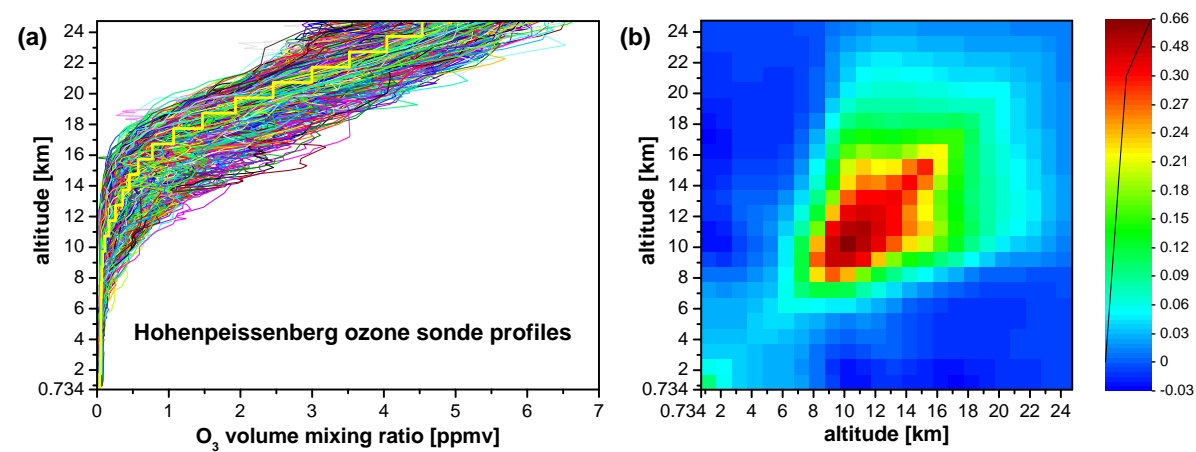

Fig. 7. Climatological ensemble of ozone sonde profiles used to construct the Zugspitze $\mathrm{O}_{3}$ a priori profile (a) and the $\mathrm{O}_{3}$ climatological covariance (b). Note, that the units of the state vector are scaling factors for the VMR-layer averages of the a priori profile given on a $1 \mathrm{~km}$ grid.
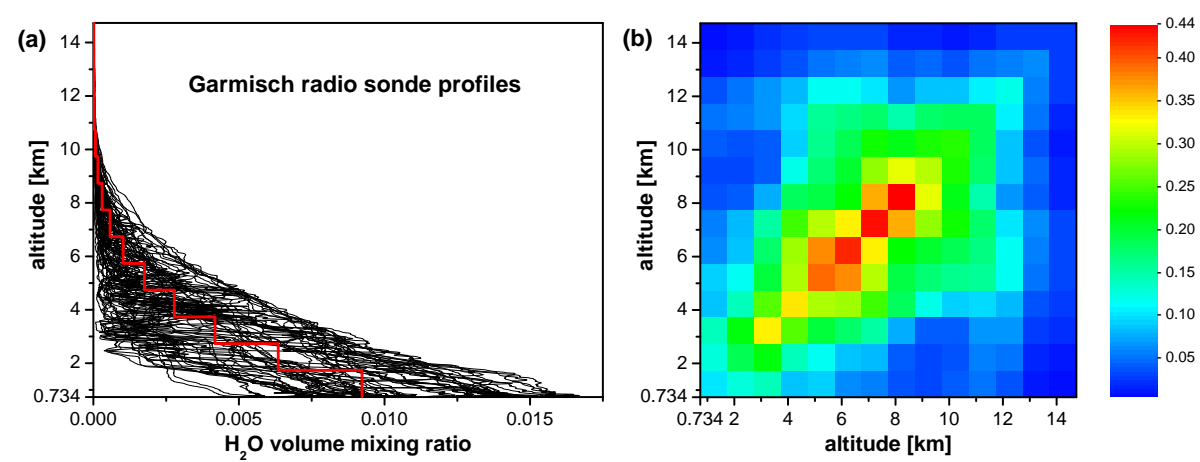

Fig. 8. Climatological ensemble of radio sonde profiles used to construct the Zugspitze $\mathrm{H}_{2} \mathrm{O}$ a priori profile (a) and the $\mathrm{H}_{2} \mathrm{O}$ climatological covariance (b). Note, that the units of the state vector are scaling factors for the VMR-layer averages of the a priori profile given on a $1 \mathrm{~km}$ grid.

July/August 2000 and May/June 2003 between $31-56^{\circ} \mathrm{N}$. This data was provided by the ETHmeg data base, see Fig. 10.

\subsubsection{Resulting interference errors for the CO standard re- trieval}

Based on the results of Sects. 3.5.1 and 3.5.2 the interference error covariances $\mathbf{S}_{\mathrm{CO}-\mathrm{O} 3}, \mathbf{S}_{\mathrm{CO}-\mathrm{H} 2 \mathrm{O}}, \mathbf{S}_{\mathrm{CO}-\mathrm{N} 2 \mathrm{O}}$, and $\mathbf{S}_{\mathrm{CO}-\mathrm{CO} 2}$ were calculated according to Eq. (9). In analogy to Fig. 5 we plotted the square roots of the diagonal elements of the interference error covariances as profiles versus altitude, see Fig. 11b for the standard retrieval (VMR-profile scaling retrieval of the interfering species) using the Rinsland et al. (2000) microwindows. Again, as for the smoothing error, we plotted the full ensemble of interference errors versus altitude calculated around each of the retrieved states of the Zugspitze test ensemble. The spread of the interference error profiles of one species is due to non-linear effects (mainly due to changing SZA) as discussed in Sect. 2.3 in general and in Sect. 3.4 for the case of smoothing errors. Figure $11 \mathrm{~b}$ illustrates a crucial result of this paper, namely, that interference errors can be significant, i.e., they can be as high as smoothing errors, or even exceed them: $\mathrm{CO}-\mathrm{O}_{3}$ interference errors frequently exceed the $\mathrm{CO}$ smoothing error in the altitude range between $\approx 14-19 \mathrm{~km}$, and $\mathrm{CO}-\mathrm{H}_{2} \mathrm{O}$ interference errors are comparable to the $\mathrm{CO}$ smoothing errors in the lower troposphere. We note that the Zugspitze is a dry site and $\mathrm{CO}-\mathrm{H}_{2} \mathrm{O}$ interference errors would be even higher for low altitude sites.

Figure 4 in Rodgers and Connor (2003) should be comparable with our Fig. 11b since both figures display interference errors and smoothing errors of the standard groundbased FTIR retrieval of CO profiles. However, the interference errors shown in Fig. 4 of Rodgers and Connor (2003) are significantly smaller than the interference errors obtained in our work (see Fig. 11b). In other words, interference errors in Fig. 4 of Rodgers and Connor (2003) are more than an order of magnitude smaller than the $\mathrm{CO}$ smoothing error, while interference errors are comparable to the smoothing error or higher in our Fig. 11b. Obviously, this underestimation of interference errors arises because interference errors were directly calculated on the coarse 1-layer grid of the standard retrieval (as explained in Sect. 7.1.2 of Rodgers and Connor, 

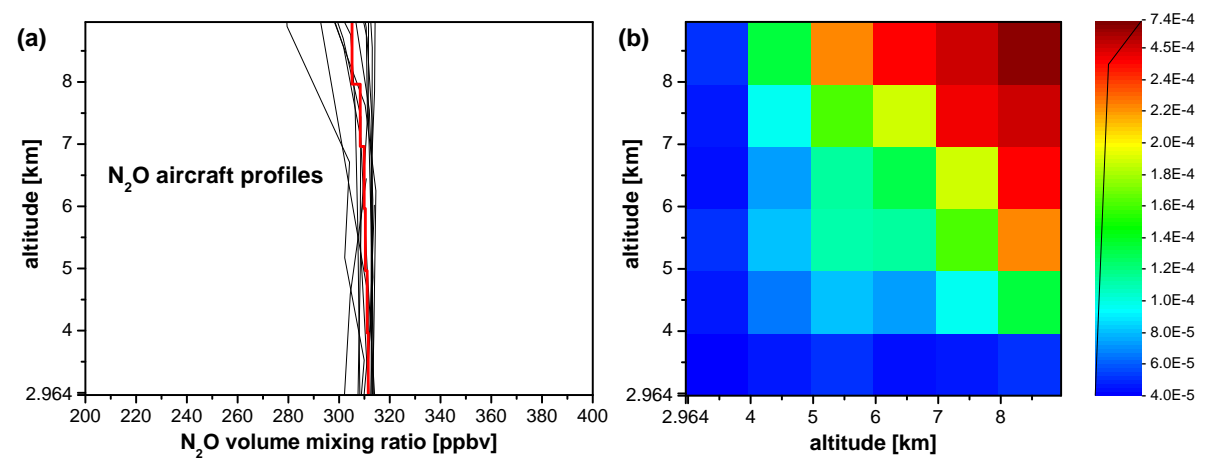

Fig. 9. Climatological ensemble of aircraft profiles used to construct the Zugspitze $\mathrm{N}_{2} \mathrm{O}$ a priori profile (a) and the $\mathrm{N}_{2} \mathrm{O}$ climatological covariance (b). Note, that the units of the state vector are scaling factors for the VMR-layer averages of the a priori profile given on a $1 \mathrm{~km}$ grid.
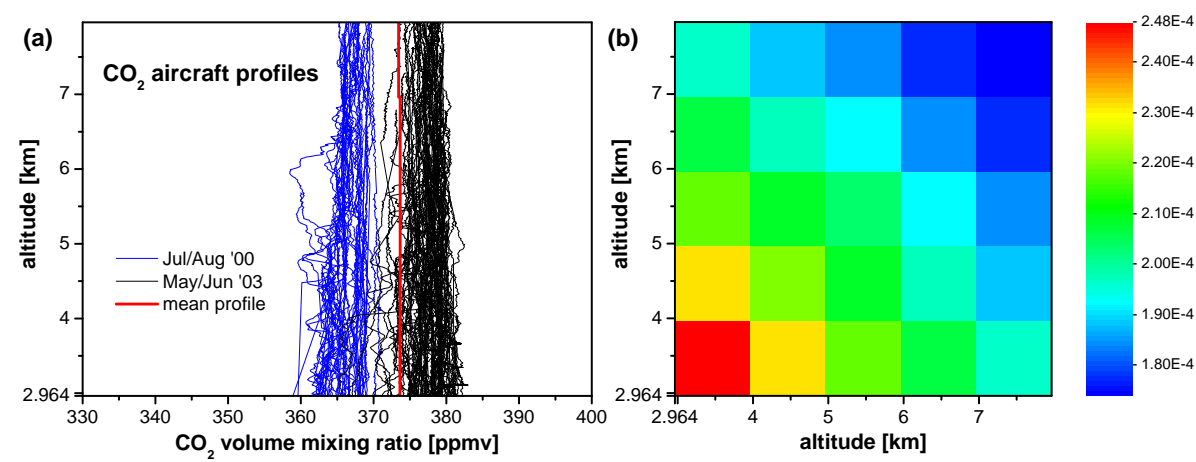

Fig. 10. Climatological ensemble of aircraft profiles used to construct the Zugspitze $\mathrm{CO}_{2}$ a priori profile (a) and the $\mathrm{CO}_{2}$ climatological covariance (b). Note, that the units of the state vector are scaling factors for the VMR-layer averages of the a priori profile given on a $1 \mathrm{~km}$ grid.

2000), and not via a fine-grid emulation of this course grid as suggested in our Sects. 2.2.2 and 3.5.1.

\section{Sensitivity studies and minimization of interference errors}

\subsection{Interference errors in case of unretrieved interfering species}

We quantify here the interference errors for the case that the interfering species are not retrieved. Since we have already implemented the interfering species within the state vector of our algorithm on a fine retrieval grid, we can quickly switch to "non-retrieval" by using our concept of "dead regularization" according to Eq. (12).

Results are shown in Fig. 11a. All interference errors have strongly increased as compared to the standard retrieval, where the interfering species are retrieved via VMR-profile scaling (Fig. 11b). This striking tendency is also documented in Table 1 via numbers, utilizing the concept of "mean errors" defined in Eq. (14): in Scenario i) (unretrieved in- terfering species) the average over the $i=1 \ldots 156$ retrieved states of our Zugspitze test ensemble $\left(\mathrm{AV}_{i}\right)$ of the "total mean error" $\bar{\sigma}_{\text {tot }, i}:=\operatorname{sqrt}\left(\bar{\sigma}_{\mathrm{CO}-\mathrm{CO}, i}^{2}+\bar{\sigma}_{\mathrm{CO}-\mathrm{O} 3, i}^{2}+\bar{\sigma}_{\mathrm{CO}-\mathrm{H} 2 \mathrm{O}, i}^{2}\right.$ $\left.+\bar{\sigma}_{\mathrm{CO}-\mathrm{N} 2 \mathrm{O}, i}^{2}+\bar{\sigma}_{\mathrm{CO}-\mathrm{CO} 2, i}^{2}\right)$ is $\mathrm{AV}_{i}\left(\bar{\sigma}_{\mathrm{tot}, i}\right)=35.23 \%$; it is dominated by a high water-vapor interference, $\mathrm{AV}_{i}$ $\left(\bar{\sigma}_{\mathrm{CO}-\mathrm{H} 2 \mathrm{O}, i}\right)=34.10 \%$. Compared with this, a VMRprofile scaling retrieval of the interfering species, reduces $\mathrm{AV}_{i}\left(\bar{\sigma}_{\mathrm{tot}, i}\right)$ from $35.23 \%$ (Scenario i) to $6.12 \%$ in Scenario ii), which is now dominated by the smoothing error $(5.21 \%)$, but is still significantly affected by interference, mainly due to $\mathrm{O}_{3}(3.12 \%)$.

\subsection{Effect from implementing the optimum strategy to so- lar $\mathrm{CO}$ retrivals}

We show now that interference errors in solar $\mathrm{CO}$ retrievals can be practically eliminated by implementing the optimum strategy for retrieval of the interfering species as introduced in Sect. 2.4.2. This means to reduce the regularization of the retrieval of the interfering species from a simple scaling retrieval (which is the standard approach) towards a weakly regularized (profile) retrieval on a fine grid. This is 

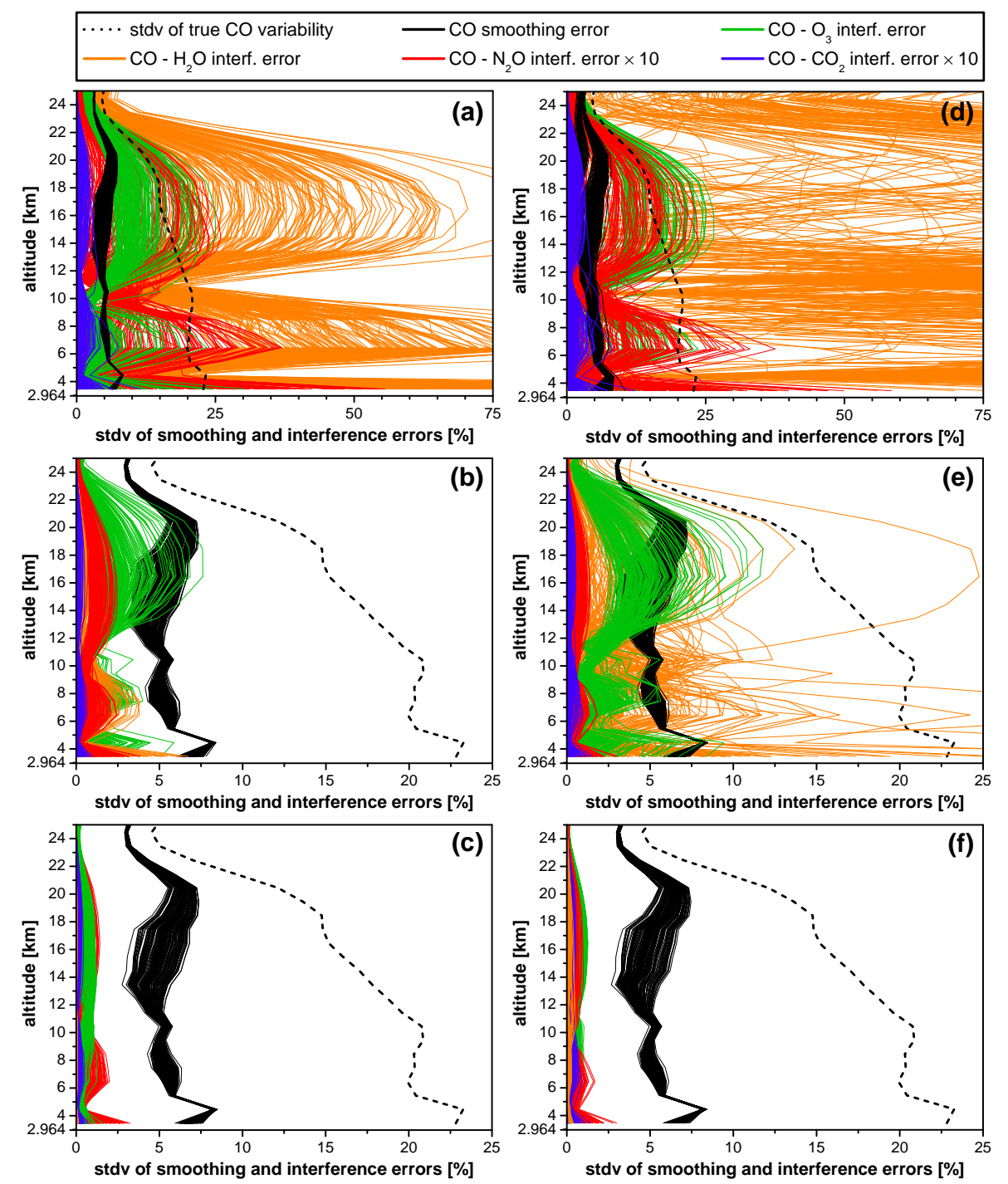

Fig. 11. Profiles of the interference errors $\mathrm{CO}-\mathrm{O}_{3}, \mathrm{CO}-\mathrm{H}_{2} \mathrm{O}, \mathrm{CO}-\mathrm{N}_{2} \mathrm{O}$, and $\mathrm{CO}-\mathrm{CO}_{2}$ (colored curves: square roots of the diagonal elements of the interference error covariances, black: same for smoothing error) calculated around all states retrieved from the Zugspitze test ensemble of 156 spectra. (a) Errors for the CO standard retrieval with the Rinsland et al. (2000) microwindows but the interfering species not retrieved. (b) Errors for the CO standard retrieval with the Rinsland et al. (2000) microwindows and VMR-profile scaling retrieval of the interfering species. (c) Rinsland et al. (2000) microwindows and optimized profile retrievals of the interfering species using the regularization parameters given in Table 2. (d) Widened microwindow (Fig. 1c) and non-retrieval of interfering species. (e) Widened microwindow (Fig. 1c) and VMRprofile scaling retrieval of interfering species. (f) Widened microwindow (Fig. 1c) and optimized profile retrievals of the interfering species using the regularization parameters given in Table 2.

accomplished by a slight increase in smoothing error, i.e., there is a tradeoff between both effects and a minimization of the combined error can be performed as explained in Sect. 2.4.2.

Figure 12 shows on its horizontal scale the transition from a VMR-profile scaling retrieval for $\mathrm{O}_{3}$ (using the $\mathbf{L}_{1}$ operator in combination with very high values for the regularization parameter, i.e., $\alpha_{\mathrm{O} 3}=10^{13}$ ) towards an essentially unregularized profile retrieval $\left(\alpha_{\mathrm{O} 3}=10^{-11}\right)$. The vertical scale in Fig. 12 shows the "mean smoothing errors" $\bar{\sigma}_{\mathrm{CO}-\mathrm{CO}, i}$ (red curves) and the "mean interference error" $\bar{\sigma}_{\mathrm{CO}-\mathrm{O} 3, i}$ (black curves) as defined in Eq. (14). These errors are calculated around all $i=1 \ldots 156$ retrieved states of the Zugspitze test ensemble as a function of $\alpha_{\mathrm{O} 3}$. The ensemble-type nature of these plots again results from the described non-linearity effects. As a result from Fig. 12 it can be seen, that the mean $\mathrm{CO}-\mathrm{O}_{3}$ interference errors decrease together with decreasing $\alpha_{\mathrm{O} 3}$ as expected. Figure 12 also shows that the mean CO smoothing errors are increasing slightly with decreasing $\alpha_{\mathrm{O} 3}$ as expected. Therefore, an optimum $\alpha_{\mathrm{O} 3}$ for the $i$-th retrieval of the interfering species $\mathrm{O}_{3}$ can be found by searching for the minimum of the combined error, i.e., 
Table 1. Mean interference errors and smoothing errors and their dependency on the constraint of the interfering species for two different microwindow sets, i.e., the Rinsland et al. (2000) ("R-2000") set (Fig. 1) and a related set with one widened microwindow including the strong water vapor line at $2158.11 \mathrm{~cm}^{-1}$ as indicated in Fig. $1 \mathrm{c} . \mathrm{AV}_{i}$ indicates the average over our test ensemble of $i=1 \ldots 156$ retrievals. For definition of $\bar{\sigma}_{\mathrm{CO}-\mathrm{CO}, i}, \bar{\sigma}_{\mathrm{CO}-\mathrm{O} 3, i}, \ldots$ see Eq. (14), $\bar{\sigma}_{\mathrm{tot}, i}$ is the quadratic superposition of the 5 individual error contributions listed above. "opt. $\alpha$ " refers to the numbers given in Table 2.

\begin{tabular}{|c|c|c|c|c|c|c|}
\hline & \multicolumn{3}{|c|}{ R-2000 microwindow set (Fig. 1) } & \multicolumn{3}{|c|}{ R-2000 with widened microwindow (Fig. 1c) } \\
\hline & $\begin{array}{l}\text { Scenario i) unre- } \\
\text { trieved interfer- } \\
\text { ing species } \\
\text { (Fig. 11a) }\end{array}$ & $\begin{array}{l}\text { Scenario ii) } \\
\text { VMR-profile } \\
\text { scaling re- } \\
\text { trieval for all } \\
\text { interfering } \\
\text { species } \\
\text { (Fig. 11b) }\end{array}$ & $\begin{array}{l}\text { Scenario iii) } \\
\text { opt. } \alpha \text { used } \\
\text { for fine-grid } \\
\text { profile re- } \\
\text { trieval of all } \\
\text { interfering } \\
\text { species } \\
\text { (Fig. 11c) }\end{array}$ & $\begin{array}{l}\text { Scenario iv) } \\
\text { unretrieved } \\
\text { interfering } \\
\text { species } \\
\text { (Fig. 11d) }\end{array}$ & $\begin{array}{l}\text { Scenario v) } \\
\text { VMR-profile } \\
\text { scaling re- } \\
\text { trieval for all } \\
\text { interfering } \\
\text { species } \\
\text { (Fig. 11e) }\end{array}$ & $\begin{array}{l}\text { Scenario vi) } \\
\text { opt. } \alpha \text { used } \\
\text { for fine-grid } \\
\text { profile re- } \\
\text { trieval of all } \\
\text { interfering } \\
\text { species } \\
\text { (Fig. 11f) }\end{array}$ \\
\hline $\mathrm{AV}_{i}\left(\bar{\sigma}_{\mathrm{CO}-\mathrm{CO}, i}\right)$ & $5.11 \%$ & $5.21 \%$ & $5.26 \%$ & $4.66 \%$ & $5.15 \%$ & $5.17 \%$ \\
\hline $\mathrm{AV}_{i}\left(\bar{\sigma}_{\mathrm{CO}-\mathrm{O} 3, i}\right)$ & $7.19 \%$ & $3.12 \%$ & $0.57 \%$ & $6.82 \%$ & $1.94 \%$ & $0.51 \%$ \\
\hline $\mathrm{AV}_{i}\left(\bar{\sigma}_{\mathrm{CO}}-\mathrm{H} 2 \mathrm{O}, i\right)$ & $34.10 \%$ & $0.72 \%$ & $0.03 \%$ & $194.04 \%$ & $2.52 \%$ & $0.16 \%$ \\
\hline $\mathrm{AV}_{i}\left(\bar{\sigma}_{\mathrm{CO}-\mathrm{N} 2 \mathrm{O}, i}\right)$ & $0.91 \%$ & $0.09 \%$ & $0.04 \%$ & $0.74 \%$ & $0.05 \%$ & $0.04 \%$ \\
\hline $\mathrm{AV}_{i}\left(\bar{\sigma}_{\mathrm{CO}}-\mathrm{CO} 2, i\right)$ & $0.06 \%$ & $0.01 \%$ & $0.01 \%$ & $0.08 \%$ & $0.01 \%$ & $0.01 \%$ \\
\hline $\mathrm{AV}_{i}\left(\bar{\sigma}_{\mathrm{tot}, i}\right)$ & $35.23 \%$ & $6.12 \%$ & $5.29 \%$ & $194.22 \%$ & $6.05 \%$ & $5.20 \%$ \\
\hline
\end{tabular}

Table 2. Optimum settings of the regularization strength $\alpha$ for retrieval of the interfering species $\mathrm{O}_{3}, \mathrm{H}_{2} \mathrm{O}, \mathrm{N}_{2} \mathrm{O}$, and $\mathrm{CO}_{2}$ found from combined minimization of interference errors and smoothing errors. Results are presented for the standard Rinsland et al. (2000) ("R-2000") mircowindows (Fig. 1) as well as for a related set with one widened microwindow including the strong water vapor line at $2158.11 \mathrm{~cm}^{-1}$ as indicated in Fig. 1c.

\begin{tabular}{lllll}
\hline & $\alpha_{\mathrm{O} 3}$ & $\alpha_{\mathrm{H} 2 \mathrm{O}}$ & $\alpha_{\mathrm{N} 2 \mathrm{O}}$ & $\alpha_{\mathrm{CO} 2}$ \\
\hline R-2000 microwindows & $10^{2}$ & $10^{0}$ & $10^{5}$ & $10^{13}$ \\
R-2000 with widened microwindow & $10^{2}$ & $10^{1}$ & $10^{5}$ & $10^{11}$ \\
\hline
\end{tabular}

$\operatorname{sqrt}\left(\bar{\sigma}_{\mathrm{CO}-\mathrm{CO}, i}^{2}\left(\alpha_{\mathrm{O} 3}\right)+\bar{\sigma}_{\mathrm{CO}-\mathrm{O} 3, i}^{2}\left(\alpha_{\mathrm{O} 3}\right)\right)$, see green curves in Fig. 12. The resulting optimum setting for $\alpha_{\mathrm{O} 3}$ is found from the minimum of the average of all green curves (blue diamonds in Fig. 12), i.e., $\alpha_{\mathrm{O} 3}=10^{2}$. The optimization for $\alpha_{\mathrm{H} 2 \mathrm{O}}$, $\alpha_{\mathrm{N} 2 \mathrm{O}}$, and $\alpha_{\mathrm{CO} 2}$ can be performed in an analogous manner; the results are summarized in Table 2.

The effect of incorporating these four optimized $\alpha$ values into the retrieval is shown in Fig. 11c. The interference errors are significantly reduced compared to the RRC standard retrieval where all interfering species were retrieved via VMR-profile scaling (Fig. 11b), and are now much smaller than the smoothing error for all altitude regions. The corresponding effect on the mean errors is shown in Table 1. Scenario iii) corresponds to Fig. 11c, i.e., using the optimum $\alpha$-values for all four interfering species. This reduces $\mathrm{AV}_{i}\left(\bar{\sigma}_{\mathrm{CO}-\mathrm{O} 3, i}\right)$ down to $0.57 \%$, which can be compared to the value of $3.12 \%$ obtained in the standard Scenario ii). At the same time, $\mathrm{AV}_{i}\left(\bar{\sigma}_{\mathrm{CO}-\mathrm{CO}, i}\right)$ increases only slightly from $5.21 \%$ to $5.26 \%$. In addition, $\mathrm{AV}_{i}\left(\bar{\sigma}_{\mathrm{CO}-\mathrm{H} 2 \mathrm{O}, i}\right)$ is decreased from $0.72 \%$ down to $0.03 \%$. The improvement of $\mathrm{AV}_{i}\left(\bar{\sigma}_{\text {tot, } i}\right)$ is from $6.12 \%$ (Scenario ii) to $5.29 \%$ (Scenario iii). We want to make the point that with Scenario iii) the interference errors have in fact been practically eliminated, since $\mathrm{AV}_{i}\left(\bar{\sigma}_{\text {tot }, i}\right)=5.29 \%$ of the optimized Scenario iii) is only marginally higher than the smoothing error $\mathrm{AV}_{i}\left(\bar{\sigma}_{\mathrm{CO}-\mathrm{CO}, i}\right)$ $=5.21 \%$ of the standard Scenario ii).

Due to the eliminated interference errors we suggest using the optimized retrieval (Scenario iii, Fig. 11c) instead of the standard RRC setup (Scenario ii, Fig. 11b). In order to minimize the computation effort, it is sufficient to retrieve $\mathrm{O}_{3}$ and $\mathrm{H}_{2} \mathrm{O}$ profiles on the fine grid, because the interference errors from $\mathrm{CO}_{2}$ and $\mathrm{N}_{2} \mathrm{O}$ are already negligible using VMR-profile scaling, see Table 1. Implementation of this optimized setup leads to a factor of $\approx 4$ increase in computation time for our algorithm. Using modern parallel processing techniques, this should present no hindrance for retrieval of long time series.

\subsection{Experiment with widened microwindow}

In the Rinsland et al. (2000) microwindow set there is only one weak water vapor feature at $2156.57 \mathrm{~cm}^{-1}$, which is nearly hidden below the strong wing of the saturated CO R3 absorption line (Fig. 1c). Therefore, we decided to investigate the effect of widening this microwindow to the range $2156.0-2159.15 \mathrm{~cm}^{-1}$, in order to include one additional, strong water line located at $2158.11 \mathrm{~cm}^{-1}$ (see grey shaded area in Fig. 1c). 
The question is whether the $\mathrm{CO}-\mathrm{H}_{2} \mathrm{O}$ interference error as well as the overall (smoothing and interference) error is decreased or increased by widening the microwindow. The answer is nontrivial, i.e., the sign of the net effect depends on the type of constraint applied to the interfering species. The details follow.

\subsubsection{Effect in the case of unretrieved interfering species}

When the interfering species are not retrieved, the $\mathrm{CO}-\mathrm{H}_{2} \mathrm{O}$ interference error is dramatically increased due to the microwindow widening: compare Fig. 11d to Fig. 11a (orange curves). Table 1 shows, that this corresponds to an increase in $\mathrm{AV}_{i}\left(\bar{\sigma}_{\mathrm{CO}-\mathrm{H} 2 \mathrm{O}, i}\right)$ from $34.10 \%$ (Scenario i) to $194.04 \%$ (Scenario iv). At the same time, $\mathrm{AV}_{i}\left(\bar{\sigma}_{\mathrm{CO}-\mathrm{CO}, \mathrm{i}}\right)$ is reduced from $5.11 \%$ (Scenario i) to $4.66 \%$ (Scenario iv) due to the widening of the microwindow. Both effects are to be expected from our basic considerations in Sect. 2.4.3. Due to the microwindow widening, $\mathrm{AV}_{i}\left(\bar{\sigma}_{\mathrm{tot}, i}\right)$ is increased from $35.23 \%$ (Scenario i) to $194.22 \%$ (Scenario iv), and it is dominated in both cases by the $\mathrm{CO}-\mathrm{H}_{2} \mathrm{O}$ interference error.

\subsubsection{Effect in the case of optimum strategy using fine-grid profile retrieval of interfering species}

In case the optimum strategy for retrieval of the interfering species (Sect. 2.4.2) is implemented to solar CO retrievals (as performed in Sect. 4.2), the $\mathrm{CO}-\mathrm{H}_{2} \mathrm{O}$ interference error is practically eliminated in comparison to the smooothing error - this holds true both for the case of the widenend microwindow (see Fig. 11f) and the original microwindow set (Fig. 11c). Table 1 shows that widening of the microwindow leads to a slightly reduced $\operatorname{AV}_{i}\left(\bar{\sigma}_{\text {tot }, i}\right)=5.20 \%$ (Scenario vi) compared to $5.29 \%$ for the original microwindows (Scenario iii). Note, that microwindow widening now has the opposite effect of that described in Sect. 4.3.1 for the case of unretrieved interfering species. This can be understood by the fact that our strategy of systematically reducing the regularization for the profile retrieval of the interfering species leads to an effective elimination of the interference effect. Therefore, an overall reduction in total (smoothing and interference) error, which is due to the fact that a widenend microwindow leads to a reduced smoothing error (5.17\% for Scenario vi versus $5.26 \%$ for Scenario iii), as expected from our basic considerations in Sect. 2.4.3.

All in all, implementation of the optimum strategy to solar $\mathrm{CO}$ retrievals practically eliminates all interference effects. In consequence, microwindow selection, which is very critical in the case of unretrieved interfering species (compare Fig. 11d with Fig. 11a) becomes uncritical (compare Fig. 11f and Fig. 11c). The maximum microwindow width is then mainly a question of available computation power (and possible forward model errors that could come into play due to the widening). In consequence, the two considerably different microwindow sets (see Fig. 1c) can be applied without

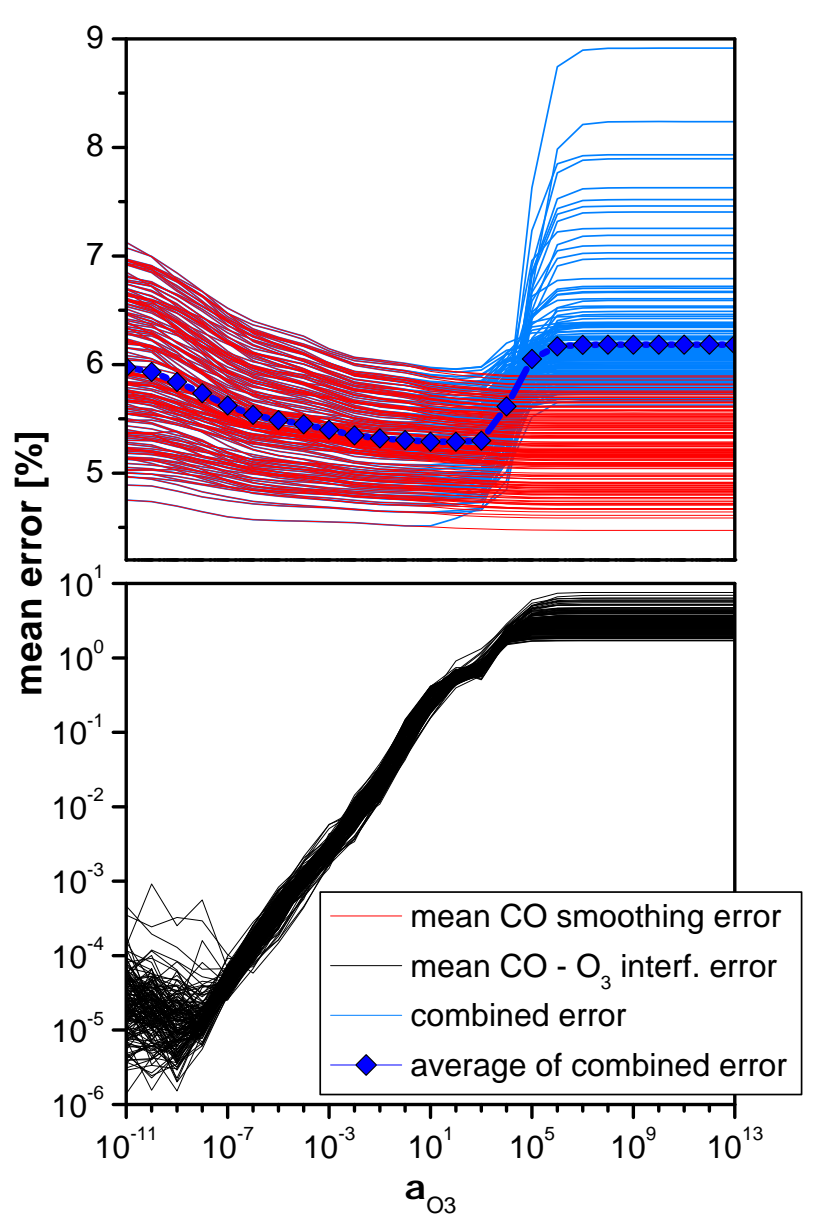

Fig. 12. This image displays the tradeoff between minimizing interference errors and the smoothing error. Plotted are altitude averaged $\mathrm{CO}$ smoothing errors (red curves) and $\mathrm{CO}-\mathrm{O}_{3}$ interference errors (black curves) calculated around all states retrieved from the 156 spectra of the Zugspitze test ensemble as a function of the regularization strength $\alpha_{\mathrm{O} 3}$. The combined $\mathrm{CO}$ smoothing and $\mathrm{CO}-\mathrm{O}_{3}$ interference errors are plotted (blue curves), and their average (blue diamonds) shows a minimum for $\alpha_{\mathrm{O} 3}=10^{2}$.

large practical differences, if the optimum strategy is implemented: there are slightly lower combined (smoothing and interference) errors for the widened microwindow, which is at the cost of somewhat increased computation time.

\subsubsection{Effect in the case of standard VMR-profile scaling retrieval of interfering species}

For the historical RRC approach utilizing VMR-profile scaling retrieval of all interfering species, the widened microwindow would be less favorable due to higher non-linear effects encountered (see higher scatter in the ensembles of error profiles in Fig. 11e compared to Fig. 11b). The ensemble average of the mean total error remains nearly unchanged, however $(6.05 \%$ versus $6.12 \%$, see Table 1$)$. 


\section{Summary and conclusions}

This paper shows that a class of potentially significant errors exists in infrared remote sounding of profiles of atmospheric composition that has not been treated by the classical error analysis of remote sounding given by Rodgers $(1990,2000)$. This new class of "interference errors" supplements the wellknow traditional classes of "smoothing errors", "model parameter errors", "forward model errors", and "retrieval noise errors". Interference errors are a concern for atmospheric spectroscopy in the infrared domain, since many vibrationrotation bands of different trace species overlap there. This is not the case in the microwave atmospheric spectrum. The interference effect physically originates from spectral residuals from interfering species which then lead to artifacts in the target species retrieval.

A general method for quantification of interference errors is given in Sect. 2. It covers all three different algorithmic implementations of interfering species, namely fine-grid profile retrievals of the interfering species (Case I), coarse-grid retrievals (Case II), and cases where the interfering species are not retrieved (Case III). Case I formulation is based on a generalized state vector comprising all retrieval parameters, including fine-grid profile retrievals of the interfering species in addition to the target species. This leads to a generalized averaging kernel matrix comprising classical averaging kernels plus newly-defined interference kernels. The latter are used for estimating the interference errors, using climatological covariances describing the true atmospheric profile variability of the interfering species.

Quantification of interference errors for Case II algorithms using coarse-grid retrieval of interfering species (e.g., via simple VMR-profile scaling), requires implementation of a fine retrieval grid for the interfering species. This is a prerequisite for mapping the true high-resolution atmospheric profile covariance of the interfering species into the error analysis properly. The coarse-grid retrieval of the interfering species used in the algorithm to be characterized then has to be emulated on the fine retrieval grid. We show how this can be achieved by using a block-Tikhonov-type first order regularization matrix (with a very high regularization strength). We made the crucial point that calculating interference errors directly on a coarse grid is erroneous, i.e., the interference errors are strongly underestimated.

In the past, operational algorithms that did not retrieve interfering species (Case III) have been used because of limited computation power. In this case interference errors could be quantified using the classical concept of model parameter errors, according to Rodgers (2000, Eq. 3.16, second term). This is somewhat laborious, since the covariance of the interfering species has to be transferred to the measurement space (via a Jacobian $\mathbf{K}_{b}$ ) and then mapped back to the state space. Therefore, we suggest a simpler alternative which formally retrieves interfering species, but uses a "dead regularization" to emulate non-retrieval of the interfering species directly in state space (Sect. 2.2.3). This approach may be useful, e.g., for a quick test of the effect of non-retrieval versus retrieval of a certain interfering species (e.g., this was performed in Sect. 4.1).

Part of our general formulation describes the fact that non-linearity of the retrieval can have a significant effect on the magnitude of interference errors and smoothing errors (Sect. 2.3). We found for the example of CO standard profile retrievals from solar FTIR, that interference errors and smoothing are in fact varying considerably from state to state, which is mainly due to the varying solar zenith angle. This implies that the common practice in the infrared community of using just one "typical" averaging kernel for calculation of smoothing errors is generally not valid. Calculation of smoothing errors and interference must then be carried out on a state-to-state basis.

The general formulation is illustrated by applying it to the example of optimal estimation of $\mathrm{CO}$ profiles from groundbased mid-infrared solar spectra recorded with the highresolution Fourier Transform spectrometer at the NDACC Primary Station Zugspitze, Germany (Sect. 3). As a crucial result from this paper, is that in the widely used standard setup employing VMR-profile scaling retrieval of the interfering species, $\mathrm{CO}-\mathrm{O}_{3}$ interference errors frequently exceed the CO smoothing error in the altitude range between $\approx 14$ $19 \mathrm{~km}$, and $\mathrm{CO}-\mathrm{H}_{2} \mathrm{O}$ interference errors are comparable to the $\mathrm{CO}$ smoothing errors in the lower troposphere.

We present an optimum strategy to practically eliminate interference errors (Sect. 2.4.2). It requires implementation of fine-grid Tikhonov-type profile retrievals for all interfering species. In consequence, the regularization strength applied to the profile retrieval for an interfering species then has to be minimized via one parameter, leading to a trade-off between decreasing interference error and slightly increasing smoothing error. A search for the minimum of total error (comprising interference error and smoothing error) yields the optimum regularization strength for retrieval of the interfering species.

Using this optimum strategy, the details of microwindow selection become uncritical as we have shown in Sects. 2.4.3 and 4.3.2. Widening of microwindow borders has then just the effect of slightly increasing the information content for the target species at the cost of increased computation time. There is no longer a significant increase of interference errors as it is the case if the interfering species are not retrieved, or if they are retrieved via VMR-profile scaling.

The optimum strategy has been applied to CO profile retrievals from solar spectra (Sect. 4.2). It has been demonstrated that changing the FTIR standard retrieval from using a VMR-profile scaling retrieval of the four interfering species to the optimum strategy using fine-grid profile retrievals for the interfering species $\mathrm{O}_{3}$ and $\mathrm{H}_{2} \mathrm{O}$ with a Tikhonov first order regularization (using $\alpha_{\mathrm{O} 3}=10^{2}, \alpha_{\mathrm{H} 2 \mathrm{O}}=10^{0}$ ), effectively eliminates all interference errors. This optimized setup cost a factor of $\approx 4$ increase in computation time. This should 
not be a hindrance, not even for routine retrievals of decadal time series, if state-of-the-art multi-node parallel processing is utilized.

The optimum strategy for retrieval of interfering species presented in this paper is based upon a minimization of combined interference errors and smoothing errors, as a function of the regularization strength used for retrieval of the interfering species. In this context it is remarkable that we could conjecture from basic considerations in Sect. 2.4.2, that application of this optimum strategy leads at the same time always to reduced forward model (parameter) errors and reduced retrieval noise.

The findings of this paper can be applied to soundings of all infrared-active atmospheric trace gases, which are more than two dozen gases with relevance to climate and ozone. In addition, these findings hold for all kinds of infrared remote sounding systems, i.e., retrievals from ground-based, balloon-borne, airborne, or satellite spectroradiometers.

In operational satellite retrievals, the interfering species have historically often not been retrieved due to computational power limitations. However, we might forecast a tendency that - as computation power increases in the future more and more satellite retrievals will be able to utilize our optimum strategy of jointly retrieving profiles of all interfering species with weak (minimized) regularization, since this practically eliminates the interference effect. Furthermore, in so doing, the issue of microwindow selection will become much less critical as has been shown and mentioned above. This is because a weakly regularized profile retrieval of interfering species represents an interference-species selective deweighting of the retrieval. This automatically takes the actual strength of the spectral feature of the interfering species into account, e.g., depending on tangent altitude. Therefore, there will be less need for extensive microwindow cutting in future satellite retrievals and the information content for the target species will increase at the same time.

In a study by Bowman et al. (2006), errors in $\mathrm{O}_{3}$ profiles from interfering retrieval of $\mathrm{H}_{2} \mathrm{O}$ and $\mathrm{T}$ profiles have been quantified for the Tropospheric Emission Spectrometer (TES). This is an example for the hitherto rare case of a joint fine-grid retrieval of interfering species in satellite remote sensing (corresponding to Case I of our general formulation), which, however, has not been optimized in the sense of our Sect. 2.4.2.

\section{Appendix A}

\section{Definitions and classical error analysis}

Our general formulation of interference errors in Sect. 2 is an extension of Roger's $(1990,2000)$ classical formulation of error analysis of remote sounding. Therefore, we briefly repeat here the Rodgers (2000) formulation and the definitions used.
According to Rodgers (2000, Eq. 3.16) the retrieved target profile $\hat{\boldsymbol{x}}$ is related to the true target profile $\boldsymbol{x}$ via the relation

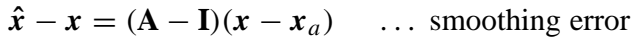

$$
\begin{aligned}
& +\mathbf{G}_{y} \mathbf{K}_{b}(\boldsymbol{b}-\hat{\boldsymbol{b}}) \quad \ldots \text { model parameter error } \\
& +\mathbf{G}_{y} \Delta \mathbf{f}\left(\boldsymbol{x}, \boldsymbol{b}, \boldsymbol{b}^{\prime}\right) \quad \ldots \text { forward model error } \\
& +\mathbf{G}_{y} \boldsymbol{\varepsilon} \quad \cdots \text { retrieval noise , }
\end{aligned}
$$

where $\mathbf{A}=\partial \hat{\boldsymbol{x}} / \partial \boldsymbol{x}, \mathbf{G}_{y}=\partial \hat{\boldsymbol{x}} / \partial \boldsymbol{y}, \mathbf{K}_{b}=\partial \mathbf{F} / \partial \boldsymbol{b}$, and $\boldsymbol{x}_{a}$ represents the a priori profile. The forward model parameters (which are not retrieved) are represented by $\boldsymbol{b}$, and $\hat{\boldsymbol{b}}$ is our best estimate of the forward model parameters, as distinct from the true value $\boldsymbol{b}$. The forward function $\mathbf{f}$ describes the true physical relation between the measurement vector $\boldsymbol{y}$ and $x$

$\boldsymbol{y}=\mathbf{f}\left(x, b, b^{\prime}\right)+\varepsilon$.

Measurement noise is described by the error term $\boldsymbol{\varepsilon}$. The forward model $\mathbf{F}$ is related to $\mathbf{f}$ via the relation

$\Delta \mathbf{f}=\mathbf{f}\left(x, b, b^{\prime}\right)-\mathbf{F}(x, b)$,

where $\boldsymbol{b}^{\prime}$ represents all forward function parameters which are ignored in the construction of $\mathbf{F}$, and $\Delta \mathbf{f}$ is the error in the forward model relative to real physics.

\section{Appendix B}

\section{Retrieval and different types of constraint}

While the forward model $\mathbf{F}$ maps from the state space (quantities to be retrieved) into the measurement space (spectrum), we are interested in the inverse mapping which is formulated as a least squares problem. Due to the non-linearity of $\mathbf{F}$, a Newtonian iteration is applied and a regularization term $\mathbf{R} \in \Re^{. n \times n}$ is used that allows one to add additional information about the solution and thereby avoid oscillating profiles

$$
\begin{aligned}
\boldsymbol{x}_{i+1}= & \boldsymbol{x}_{i}+\left(\mathbf{K}_{x, i}^{T} \mathbf{S}_{\varepsilon}^{-1} \mathbf{K}_{x, i} \mathbf{R}\right)^{-1} \\
& \times\left\{\mathbf{K}_{x, i}^{T} \mathbf{S}_{\varepsilon}^{-1}\left[\boldsymbol{y}-\mathbf{F}\left(\boldsymbol{x}_{i}\right)\right]-\mathbf{R}\left(\boldsymbol{x}_{i}-\boldsymbol{x}_{a}\right)\right\}
\end{aligned}
$$

where the subscript $i$ denotes the iteration index (see Appendix A for definitions).

In the following we present two different types of $\mathbf{R}$, i.e., optimal estimation (Rodgers, 1976) and Tikhonov regularization (Tikhonov, 1963).

In the case of optimal estimation, $\mathbf{R}$ is setup using the relation

$\mathbf{R}=\mathbf{S}_{R}^{-1}$,

where $\mathbf{S}_{R} \in \Re^{n \times n}$ is the a priori covariance matrix. In the ideal case $\mathbf{S}_{R}$ is a climatological covariance constructed from an ensemble of true profiles covering the full range of possible atmospheric states. We use optimal estimation in this paper for the retrieval of profiles of the target species $\mathrm{CO}$. 
In the case of Tikhonov regularization, $\mathbf{R}$ is setup using the relation

$\mathbf{R}=\alpha \mathbf{L}^{T} \mathbf{L}$,

where $\alpha$ is the strength of the constraint and $\mathbf{L}$ is the constraint operator. For example, the discrete first derivative operator $\mathbf{L}_{1}$

$\mathbf{L}_{1}=\left(\begin{array}{ccccc}-1 & 1 & 0 & \ldots & 0 \\ 0 & -1 & 1 & \ddots & \vdots \\ \vdots & \ddots & \ddots & \ddots & 0 \\ 0 & \ldots & 0 & -1 & 1\end{array}\right) \in \Re \quad n \times(n-1)$

constrains the difference between the retrieved profile and the a priori profile. This means that the $\mathbf{L}_{1}$ operator constrains the shape of the retrieved profile but not its absolute values which are determined by the measurement. While means to optimize the magnitude of $\alpha$ for various purposes have been given by Steck (2002) in detail, we will restrict our discussion to two limiting cases.

The case $\alpha \rightarrow \infty$ represents an infinitely strong soft constraint to the profile shape and a zero constraint to the absolute value of the profile scaling factor. Therefore we can use this type of soft constraint to emulate a 1-layer retrieval grid (e.g., VMR-profile scaling) on a fine grid. This idea is extended to multi-layer coarse grids in Sect. 2.2.2. This kind of emulation is a pre-requisite for quantification of interference errors in case the interfering species are retrieved on a coarse grid (see Sect. 2.2.2 for details).

The case $\alpha \rightarrow 0$ describes a retrieval without any regularization. In consequence, the retrieved profiles will frequently suffer from oscillations, but there will be a perfect fit (no spectral residual). Therefore this kind of zero regularization - if applied to the retrieval of interfering species - eliminates the interference error. This effect is utilized in Sect. 2.4.2.

Acknowledgements. We thank W. Seiler (IMK-IFU) for his continual interest in this work, and W. Stremme (IMK-IFU) for valuable scientific discussions. We would also like to thank H. Claude (German Weather Service) for providing the Hohenpeissenberg ozone sonde data and D. Brunner (EMPA) for making available aircraft profiles via access to the ETHmeg data. Basic funding of the NDACC Primary Station Zugspitze/Garmisch and Permanent Ground-Truthing Facility Zugspitze/Garmisch has been provided by the German Federal Ministry of Education and Research (BMBF) via the Program "Atmosphere and Climate" of the German Helmholtz Association of National Research Centres. Funding by the EC within the projects UFTIR (contract EVK2-CT-2002-00159) and HYMN (contract GOCE 037048) is gratefully acknowledged. This work contributes to the ESA-ENVISAT-Validation-Project TASTE and is part of the EC-Network of Excellence ACCENT-TROPOSAT-2.

Edited by: U. Pöschl

\section{References}

Bowman, K., Rodgers, C. D., Kulawik, S. S., Worden, J., Sarkissian, E., Ostermann, G., Steck, T., Lou, M., Eldering, A., Shephard, M., Worden, H., Lampel, M., Clough, S., Brown, P., Rinsland, C., Gunson, M., and Beer, R.: Tropospheric Emission Spectrometer: Retrieval Method and Error Analysis, IEEE Trans. Geosci. Remote Sens., 44, 1297-1307, 2006.

Connor, B. J., Parrish, A., Tsou, J.-J., and McCormick, M. P.: Error analysis for the ground-based microwave ozone measurements during STOIC, J. Geophys. Res., 100, 9283-9291, 1995.

Deeter, M. N., Emmons, L. K., Francis, G. L., Edwards, D. P., Gille, J. C., Warner, J. X., Khattatov, B., Ziskin, D., Lamarque, J.-F., Ho, S.-P., Yuding, V., Attie, J.-L. Packman, D., Chen, J., Mao, D., and Drummond, J. R.: Operational carbon monoxide retrieval algorithm and selected results for the MOPITT instrument, J. Geophys.Res., 108, 4399-4409, 2003.

Dudhia, A., Jay, V. L., and Rodgers, C. D.: Microwindow selection for high-spectral-resolution sounders, Appl. Opt., 41, 36653673, 2002.

Echle, G., von Clarmann, T., Dudhia, A., Flaud, J.-M., Funke, B., Glatthor, N., Kerrridge, B., López-Puertas, M., Martín-Torres, F. J., and Stiller, G.: Optimized spectral microwindows for data analysis of the Michelson Interferometer for Passive Atmospheric Sounding on the Environmental Satellite, Appl. Opt., 39, 5531-5540, 2000.

Jones, N. B., Rinsland, C. P., Liley, J. B., and Rosen, J.: Correlation of aerosol and carbon monoxide at $45^{\circ} \mathrm{S}$ : Evidence of biomass burning emissions, Geophys. Res. Lett., 28, 709-712, 2001.

Paton-Walsh, C., Jones, N. B., Wilson, S. R., Haverd, V., Meier, A., Griffith, D. W. T., and Rinsland, C. P.: Measurements of trace gas emissions from Australian forest fires and correlations with coincident measurements of aerosol optical depth, J. Geophys. Res., 110, D24305, 2005, doi:10.1029/2005JD006202.

Pougatchev, N. S. and Rinsland, C. P.: Spectroscopic study of the seasonal variation of carbon monoxide vertical distribution above Kitt Peak, J. Geophys. Res., 100, 1409-1416, 1995.

Pougatchev, N. S., Jones, N. B., Connor, B. J., Rinsland, C. P., Becker, E., Coffey, M. T., Connors, V. S., Demoulin, P., Dzhola, A. V., Fast, H., Grechko, E. I., Hannigan, J. W., Koike, M., Kondo, Y., Mahieu, E., Mankin, W. G., Mittermeier, R. L., Notholt, J., Reichle Jr., H. G., Sen, B., Steele, L. P., Toon, G. C., Yurganov, L. N., Zander, R., and Zhao, Y.: Ground-based infrared solar spectroscopic measurements of carbon monoxide during 1994 Measurement of Air Pollution from Space flights, J. Geophys. Res., 103(D15), 19317-19325, 1998.

Rinsland, C. P., Jones, N. B., Connor, B. J., Logan, J. A., Pougatchev, N. S., Goldman, A., Murcray, F. J., Stephen, T. M., Pine, A. S., Zander, R., Mahieu, E., and Demoulin, P.: Northern and southern hemisphere ground-based infrared spectroscopic measurements of tropospheric carbon monoxide and ethane, J. Geophys. Res., 103, 28 197-28 217, 1998.

Rinsland, C. P., Mahieu, E., Zander, R., Demoulin, P., Forrer, J., and Buchmann, B.: Free tropospheric $\mathrm{CO}, \mathrm{C}_{2} \mathrm{H}_{6}$, and $\mathrm{HCN}$ over central Europe: Recent measurements from the Jungfraujoch station including the detection of elevated columns during 1998, J. Geophys. Res., 105, 24 235-24 249, 2000.

Rinsland, C. P., Jones, N. B., Connor, B. J., Wood, S. W., Goldman, A., Stephen, T. M., Murcray, F. J., Chiou, L. S., Zander, R., and Mahieu, E.: Multilayer infrared solar spectroscopic mea- 
surements of $\mathrm{HCN}, \mathrm{CO}, \mathrm{C}_{2} \mathrm{H}_{6}$, and $\mathrm{C}_{2} \mathrm{H}_{2}$ tropospheric columns above Lauder, New Zealand ( $45^{\circ} \mathrm{S}$ latitude), J. Geophys. Res., 107(D14), 4185, doi:10.1029/2001JD001150, 2002.

Rodgers, C. D.: Retrieval of Atmospheric Temperature and Composition From Remote Measurements of Thermal Radiation, Rev. Geophys. Space Phys., 14, 609-624, 1976.

Rodgers, C. D.: Characterization and Error Analysis of Profiles Retrieved From Remote Sounding Measurements, J. Geophys. Res., 95, 5587-5595, 1990.

Rodgers, C. D.: Inverse Methods for Atmospheric Sounding: Theory and Practice, vol. 2 of Series on Atmospheric, Oceanic and Planetary Physics, edited by: Taylor, F. W., World Scientific, 2000.

Rodgers, C. D. and Connor, B. J.: Intercomparison of remote sounding instruments, J. Geophys. Res., 108(D3), 4116, doi:10.1029/2002JD002299, 2003.

Rothman, L. S., Jacquemart, D., Barbe, A., Benner, D. C., Birk, M., Brown, L. R., Carleer, M. R., Chackeria Jr., C., Chance, K., Coudert, L. H., Dana, V., Devi, V. M., Flaud, J.-M., Gamache, R. R., Goldman, A., Hartmann, J.-M., Jucks, K. W., Maki, A. G., Mandin, J.-Y., Massie, S. T., Orphal, J., Perrin, A., Rinsland, C. P., Smith, M. A. H., Tennyson, J., Tolchenov, R. N., Toth, R. A., Vander Auwera, J., Varanasi, P., and Wagner, G.: The HITRAN 2004 molecular spectroscopic database, J. Quant. Spectrosc. Radiat. Transfer, 96, 139-204, 2005.

Steck, T. and von Clarmann, T.: Constrained profile retrieval applied to the observation mode of the Michelson Interferometer for Passive Atmospheric Sounding, Appl. Opt., 40, 3559-3571, 2001.

Steck, T.: Methods for determining regularization for atmospheric retrieval problems, Appl. Opt., 41, 1788-1797, 2002.

Sussmann, R. and Schäfer, K.: Infrared spectroscopy of tropospheric trace gases: combined analysis of horizontal and vertical column abundances, Appl. Opt., 36, 735-741, 1997.

Sussmann, R. and Buchwitz, M.: Initial validation of ENVISAT/SCIAMACHY columnar CO by FTIR profile retrievals at the Ground-Truthing Station Zugspitze, Atmos. Chem. Phys., 5, 1497-1503, 2005, http://www.atmos-chem-phys.net/5/1497/2005/.

Sussmann, R., Stremme, W., Buchwitz, M., and de Beek, R.: Validation of ENVISAT/SCIAMACHY columnar methane by solar FTIR spectrometry at the Ground-Truthing Station Zugspitze, Atmos. Chem. Phys., 5, 2419-2429, 2005a.

Sussmann, R., Stremme, W., Burrows, J. P., Richter, A., Seiler, W., and Rettinger, M.: Stratospheric and tropospheric $\mathrm{NO}_{2}$ variability on the diurnal and annual scale: a combined retrieval from ENVISAT/SCIAMACHY and solar FTIR at the Permanent Ground-Truthing Facility Zugspitze/Garmisch, Atmos. Chem. Phys., 5, 2657-2677, 2005b.
Tikhonov, A.: On the solution of incorrectly stated problems and a method of regularization, Dokl. Acad. Nauk SSSR, 151, 501504, 1963.

Velazco, V., Notholt, J., Warneke, T., Lawrence, M., Bremer, H., Drummond, J., Schulz, A., Krieg, J., and Schrems, O.: Latitude and altitude variability of carbon monoxide in the Atlantic detected from ship-borne Fourier transform spectrometry, model, and satellite data, J. Geophys. Res., 110, D09306, doi:10.1029/2004JD005351, 2005.

von Clarmann, T. and Echle, G.: Selection of optimized microwindows for atmospheric spectroscopy, Appl. Opt., 37, 7661-7669, 1998.

Worden, J., Kulawik, S. S., Shephard, M. W., Clough, S. A., Worden, H., Bowman, K., and Goldman, A.: Predicted errors from tropospheric emission spectrometer nadir retrievals from spectral window selection, J. Geophys. Res., 109, D09308, doi:10.1029/2004JD00452, 2004.

Yurganov, L. N., Blumenstock, T., Grechko, E. I., Hase, F., Hyer, E. J., Kasischke, E. S., Koike, M., Kondo, Y., Kramer, I., Leung, F.-Y., Mahieu, E., Mellqvist, J., Notholt, J., Novelli, P. C., Rinsland, C. P., Scheel, H. E., Schulz, A., Strandberg, A., Sussmann, R., Tanimoto, H., Velazco, V., Zander, R., and Zhao, Y.: A quantitative assessment of the 1998 carbon monoxide emission anomaly in the Northern Hemisphere based on total column and surface concentration measurements, J. Geophys. Res., 109, D15305, doi:10.1029/2004JD004559, 2004.

Yurganov, L. N., Duchatelet, P., Dzhola, A. V., Edwards, D. P., Hase, F., Kramer, I., Mahieu, E., Mellqvist, J., Notholt, J., Novelli. P. C., Rockmann, A., Scheel, H. E., Schneider, M., Schulz, A., Strandberg, A., Sussmann, R., Tanimoto, H., Velazco, V., Drummond, J. R., and Gille, J. C.: Increased Northern Hemispheric carbon monoxide burden in the troposphere in 2002 and 2003 detected from the ground and from space, Atmos. Chem. Phys., 5, 563-573, 2005, http://www.atmos-chem-phys.net/5/563/2005/.

Zhao, Y., Kondo, Y., Murcray, F. J., Liu, X., Koike, M., Kita, K., Nakajima, H., Murata, I., and Suzuki, K.: Carbon monoxide column abundances and tropospheric concentrations retrieved from high resolution ground-based infrared solar spectra at $43.5^{\circ} \mathrm{N}$ over Japan, J. Geophys. Res., 102(D19), 23 403-23 411, 1997. 